NIKHEF/2010-30

IFF-FM-2010/02

September 2010

\title{
Asymmetric Gepner Models II. Heterotic Weight Lifting
}

\author{
B. Gato-Rivera ${ }^{a, b}$ ! and A.N. Schellekens ${ }^{a, b, c}$ \\ ${ }^{a}$ NIKHEF Theory Group, Kruislaan 409, \\ 1098 SJ Amsterdam, The Netherlands \\ ${ }^{b}$ Instituto de Física Fundamental, CSIC, \\ Serrano 123, Madrid 28006, Spain \\ ${ }^{c}$ IMAPP, Radboud Universiteit, Nijmegen, The Netherlands
}

\begin{abstract}
A systematic study of "lifted" Gepner models is presented. Lifted Gepner models are obtained from standard Gepner models by replacing one of the $\mathrm{N}=2$ building blocks and the $E_{8}$ factor by a modular isomorphic $N=0$ model on the bosonic side of the heterotic string. The main result is that after this change three family models occur abundantly, in sharp contrast to ordinary Gepner models. In particular, more than 250 new and unrelated moduli spaces of three family models are identified. We discuss the occurrence of fractionally charged particles in these spectra.
\end{abstract}

${ }^{1}$ Also known as B. Gato 


\section{Introduction}

In a previous paper [1] an analysis of spectra of simple current invariants of Gepner models [2] was presented, completing work that was initiated in 1989 in [3]. The motivation for this new, extended analysis were the statistical results on the number of families in orientifold models. The observed number of families, three, turned out to occur two to three orders of magnitude less frequently [4, 5] than the number one, two or four. We wanted to verify if the same suppression also occurred in heterotic models.

This return to the heterotic string forced us to face the problem which led one of us to essentially abandon the subject after 1989: the appearance of particles with fractional electric charge in the spectrum, an undesirable feature first observed in a class of $(2,2)$ geometric compactifications [6, 7], and then generally proved to occur for all $(0,2)$ compactifications with the standard $S U(3) \times S U(2) \times U(1)$ embedding [8] based on exact CFT constructions.

The results of [1] can be summarized as follows. No substantial progress was made on the issue of family numbers, despite a huge enlargement of the class considered: as before, three families only occur for the exceptional model usually denoted as $\left(1,16^{*}, 16^{*}, 16^{*}\right)$ [9], and for any other exceptional or non-exceptional combination the number of families is always even (see also [10]). Hence it appeared that also in the heterotic case, three families is a rare feature. On fractional charges a somewhat more optimistic conclusion was reached, namely that they are fairly often vector-like. Within the context of RCFT (rational conformal field theory) that is about the best one can reasonably hope for.

However, this analysis led to a new idea, presented in [11], and called "Heterotic Weight Lifting". Essentially this enables us to go one step further away from the lamppost of heterotic $(2,2)$ models, and get closer towards genuine $(0,2)$ models. In [3] this was done by not imposing the equivalent of space-time supersymmetry in the bosonic sector of the heterotic string; in [1] the world-sheet supersymmetry constraints between CFT building blocks were relaxed in the bosonic sector, and furthermore the $S O(10)$ group was broken (see also [12]). Heterotic weight lifting allows us to replace a minimal $\mathrm{N}=2$ model plus the $E_{8}$ gauge group in the bosonic sector by a new CFT that has exactly the same modular $S$ and $T$ matrices. This implies that modular invariance is manifestly preserved. However, the spectrum is drastically changed: the conformal weight of all ground states is shifted by integers, and the dimension of these ground states is changed as well. Because the weight of most ground states is moved up, we called this procedure "weight lifting". However, there are also some ground states that move down. Therefore, in order to be able to compute massless spectra of lifted Gepner models it is essential to be able to compute the exact spectra of standard Gepner models. This is indeed possible in the constructions considered here. In [11 a few examples of lifted Gepner models were presented, which indicated in particular that the chances for getting three families looked better. Here we want to present a complete analysis of all 435 cases of heterotic weight lifting one obtains by applying the list of lifts shown in [1] to the 168 Gepner models. We will focus on the same two issues considered in [1]: the number of families and the presence of fractional charges. On the first issue our conclusion is much more positive than the 
one in our previous paper, whereas on the second issue the conclusion remains essentially unchanged.

This paper is organized as follows. In the next section we explain our motivations. Readers who are already sufficiently motivated may skip this section, but our perspective of string phenomenology is rather different from that of others working in this area, and so we believe some explanation may be useful. In section three we summarize the heterotic weight lifting procedure. In section four we present our results on charge quantization and in section five the results on the number of families. Section six contains results on distributions of vector-like particles. In section seven we investigate whether matter in this class of models generically couples to the extra non-abelian groups provided by the heterotic weight lifting procedure, instead of the familiar $E_{8}$ factor. Finally, in section eight we formulate our conclusions.

\section{Motivation}

This paper is about RCFT, and by their nature such constructions are more suited for studying the structure of the standard model (gauge groups and representations) than for parameters and moduli. Obviously the first question to ask regarding the standard model structure is: can one build exact string theories that have a gauge group $S U(3) \times$ $S U(2) \times U(1)$, three families, and no fractionally charged exotics? It has been amply demonstrated that the answer to this question is positive, both in the case of orientifolds and heterotic strings (see our previous paper [1] for references). The common attitude in string phenomenology seems to be that this is all we need to know about the standard model structure, and that we should move on to issues like parameter values and moduli stabilization. Clearly that is indeed important. But there are other questions regarding the standard model structure we should not ignore.

A couple of decades ago, it was hoped that string theory would determine the standard model uniquely. Already very soon that turned out to be unrealistic, and it was replaced by the hope that some mysterious missing selection principle would uniquely select the standard model. Furthermore it was hoped that while waiting for the discovery of that principle, one could at least try to make a complete enumeration of all candidate vacua, and find the one that agrees with all available data, in particular the standard model and all of its parameters. Having identified it, a plethora of predictions would be available, and the correctness of the theory would be proved unambiguously by testing those predictions.

This point of view - although rarely expressed so explicitly - could still be maintained until the beginning of this century, but it seems now unlikely that we will be in that situation in the foreseeable future, because the amount of string vacua is likely to vastly exceed the currently available amount of experimental data (since the latter increases monotonically, this balance may tip in the future). One may still hope that there is something fundamentally wrong with our understanding of moduli stabilization, supersymmetry or the cosmological constant, and that we eventually will end up with a much smaller set. One may also hope that the large numbers are somehow irrelevant to the problem at hand 
(i.e. getting the standard model from string theory), and that they can be cleanly factored out from the problem, contributing only to the tuning of the cosmological constant. Even if that were true, we are still facing the problem that the methods we use to explore the string theory landscape are primitive and limited. On the exact CFT side they are mostly limited to non-interacting CFT's (free bosons, free fermions and orbifolds), a very special case, whereas geometric approaches to heterotic strings are constrained to the neighbourhood of $(2,2)$ models. In other words, at present the chances of finding "the standard model" in string theory and make exact predictions based on it seem very small. A possibly achievable goal is to prove that the landscape is dense enough in all relevant directions to contain the standard model (and anything discovered beyond it at a given time), and perhaps this is all that can be done in the foreseeable future.

But one may still hope to be able to make generic predictions for classes of models. Possible examples are extra $U(1)$ 's, rank-2 tensors that are ubiquitous in orientifold models, mirror fermions or particles with fractional electric charge. Such predictions based on generic features of certain classes will never be absolutely falsifiable, but if such particles are found they will nevertheless boost the confidence in the theory. Conversely, if it can be shown that certain particles are generically present in string theory, but are never found, one should start having serious doubts about either string theory in general, or specific classes of string theories. However, before making such predictions, it would be prudent to check if similar ones would have worked in the past. For example, had there not already been strong limits on fractionally charged particles a few decades ago, the early results of heterotic strings might have led to predict their existence. This prediction, which is actually a postdiction, would have failed. One should be highly skeptical of predictions if the postdictions are wrong. In other words, one should not only demand that the standard model is among the string vacua in a class, but also that its observed features are not excessively rare in that class. If indeed fractional charges are abundant in most heterotic string vacua that otherwise produce the standard model correctly, then this should be viewed as a serious problem.

Most of string phenomenology is focusing on getting the observed features out while ignoring the neighbouring landscape. We would argue that scanning the landscape in the neighbourhood of the standard model is essential in order to have any confidence in the correctness of a certain approach. If certain features systematically come out wrong, and can be gotten right only in very special and rare conditions, then we must assume that there is something missing in our understanding. Such failures may be due to a number of causes. Perhaps one is looking at the wrong kind of string theory (orientifolds, heterotic strings, F-theory) or one is seeing artifacts of a computation method (e.g. free fields or RCFT), or one uses a too severe approximation to a true string vacuum (for example unbroken supersymmetry or unstabilized moduli). It is also possible that certain rare features are needed for the existence of (intelligent) life, and hence it is not surprising that we observe them. If all this fails one can always claim that apparently we ended up in a universe that has some exceptionally rare features for reasons that we cannot figure out. In our opinion this is the least attractive option. In any case it is important to confront these problems, rather than ignoring them by focusing solely on string vacua that fit the 
observations.

Anthropic arguments, in the sense defined above, are unavoidable and perfectly acceptable in the context of a landscape [13, 14, 15, 16], if indeed it can be convincingly demonstrated that a certain feature is or is not important for the existence of life. But usually such arguments are just too complicated to be used reliably. What one should do instead, before worrying about a rare feature, is to try to be as certain as possible that at least anthropic arguments are not likely to make a difference. In [1] we discussed this issue for the number of families and the presence of fractionally charged particles: although it cannot be demonstrated rigorously, it is hard to believe that the existence of life requires three families (as opposed to two or four) or is inhibited by the mere existence of very rare, and heavy, fractionally charged particles (on the other hand, if they are either light or abundant the argument is far too complicated to arrive at decisive general conclusions). We would be perfectly happy to be convinced otherwise, but we take as our working hypothesis that anthropic arguments do not play a rôle here.

Any study of distributions of standard model properties is plagued by bias and measure problems (see for example [17]). Some features of distributions may be artifacts of the class considered or the method used. Furthermore distributions can be affected by factors beyond current knowledge, such as cosmological selection or moduli stabilization. Bias effects can partly be avoided by investigating the same issues using different methods or in different regions of the landscape. For example, the paucity of three family models in orientifold constructions was observed both in Gepner orientifolds and in $\mathbf{Z}_{2} \times \mathbf{Z}_{2}$ orientifolds; some questions raised in the present paper have also been examined in the context of free fermion models; and the present paper itself examines if earlier results on Gepner models hold also in a different class of heterotic models. Any investigation of distributions makes the implicit assumption that unknown factors are smoothly behaved in the relevant region of parameter space. For example, there is no reason to believe that cosmological selection or moduli stabilization favors three families, so it is difficult to believe that such effects will lift the large dip in orientifold distributions. As long as no such argument is found, the problem should be taken seriously, and not simply dismissed as a statistical fluke.

It is a matter of taste what one defines as "rare" or "unnatural". In the literature, undesirable features at the $1 \%$ level are either ignored or stressed, depending on the point of view one wishes to express. Most people would perhaps not worry about a dip of a factor of hundred in the family distribution of orientifold models, precisely at the value three. But on the other hand, most people would say that a class of models predicts a certain property if $99 \%$ of the set had that property. Likewise, convergence of gauge couplings with a precision at the $1 \%$ level is considered strong evidence by some, and ignored by others. The discrepancy of the GUT scale and the string scale by two orders of magnitude, on the other hand, is considered small by adepts of the heterotic string, and taken to infinity in discussions on F-theory GUTs. 


\subsection{Remarks on vector-like particles}

Essentially all string spectra that produce the known particles contain large numbers of additional particles: singlets, mirror quarks and leptons, higher rank tensors (in orientifold models) and in some cases even more exotic ones such as fractionally charged particles. It is reasonable to require that all additional particles should be vector-like 2 and that all matter that is chiral with respect to $S U(3) \times S U(2) \times U(1)$ has already been observed. String theory provides robust chiral spectra modulo non-robust vector-like states. To identify potential string vacua that match our world, we have to be able to match at least the robust part of the spectra.

However, it has become standard practice in string phenomenology to go much further and also match the non-robust part of the spectrum, as if we are already certain that the currently known spectrum (plus the Higgs and all superpartners) is all that exists. In other words, one assumes the absence of any vector-like particles other than those mentioned. This is a remarkable assumption given that, on the one hand, some vector-like particles are expected to exist, but on the other hand we do not (yet) have experimental information about any of them.

Exact RCFT constructions are more seriously affected by the problem of massless vector-like particles because they cannot be continuously deformed in any practical way. However, usually one gets a large number of discrete points in what appears to be the same moduli space. This results in a discrete scan over possible masses of vector-like particles, which manifests itself in the form of discrete distributions of the number of massless vector-like particles. This phenomenon has been observed both in orientifold models and heterotic models. If one is lucky enough the set includes points where certain vectorlike particles are completely absent. However, it is unlikely that the problem is solved entirely within the context of RCFT. This is simply not the right tool for solving this problem. Several steps are needed to move from the exact RCFT to a phenomenologically acceptable string vacuum, and masses for vector-like particles may be generated at any of these steps. They may acquire masses from simply moving away from the special RCFT point into the full moduli space, from moduli stabilization or from supersymmetry breaking. What is known about this is mainly folklore, based on common beliefs and sporadic examples (see e.g. [18, 19, 20, 21] for discussions of decoupling of exotic states in various models).

Let us assume, for simplicity, a scenario where the physics of moduli stabilization is cleanly separated from the physics of supersymmetry breaking, and where the latter scale is low, in the LHC range. It is quite generally accepted that all vector-like particles will acquire a mass after supersymmetry breaking (as well as the breaking of any other symmetries beyond $S U(3) \times S U(2) \times U(1))$. It is less clear what a generic string spectrum looks like when all moduli have been stabilized but supersymmetry is unbroken. If generically such a spectrum contains massless vector-like states beyond the MSSM, then this implies a generic prediction of the existence of such vector-like states with masses

\footnotetext{
${ }^{2}$ With a slight abuse of terminology (since we apply the term also to bosons) we will call all particles that can get a mass without breaking $S U(3) \times S U(2) \times U(1)$ "vector-like".
} 
generated by supersymmetry breaking, in addition to the squarks, the sleptons and the gauginos. If the SUSY scale is low, these particles should be observed at the LHC. In this scenario, it makes little sense to focus on the rare cases where such particles are absent from the exact spectrum, because the chance that these cases are realized in our universe is very small. On the other hand, if the generic situation is that vector-like particles acquire large masses in generic points in moduli space or in moduli-stabilized vacua, it is pointless to worry about their presence in exact RCFT. Many other scenarios with a variety of intermediate scales can be envisaged, but the conclusion remains unchanged in all cases where the supersymmetry breaking scale is low. If supersymmetry breaking is the last safety net to catch such particles, one would not expect it to be empty, unless something else catches them at an earlier stage.

Running of coupling constants does impose some limits on light vector-like particles, even if they cannot (yet) be observed directly. In exact string spectra there are often so many that if we extrapolate the low energy gauge couplings they develop Landau poles below the Planck scale; conversely, starting at the Planck scale they would run to far too small values in the infrared. Furthermore vector-like particles might ruin standard gauge coupling convergence, if they are not in complete GUT multiplet:3. The observation of low energy couplings roughly in agreement with the SUSY-GUT scenario does indeed eliminate many candidate heterotic string spectra, but none of these arguments completely forbids the existence of light vector-like particles, and they might even have the virtue of helping to reduce the notorious GUT-scale/string scale gap.

However, light (e.g. near the $\mathrm{TeV}$-scale) fractionally charged particles are almost certainly a phenomenological disaster, since they would be stable. The upper limit on their abundance on Earth is far less than $10^{-20}$ per nucleon [22], and stable light particles would be produced far too copiously in the early universe to be consistent with that limit [7]. Hence if we allow fractionally charged particles as vector-like massless particles in exact RCFT, we are making the implicit assumption that in the full theory they acquire essentially Planck scale, or at least GUT scale masses (the lower limit depends on various assumptions about the cosmological evolution of the universe). Provided this is what generically happens, vector-like fractional charges are acceptable in an exact RCFT spectrum. At least one can give them the benefit of the doubt.

If, on the other hand, generically some vector-like states remain light, this produces an interesting dilemma for heterotic strings. First of all one would be forced to look for examples with a rare feature, i.e. complete absence of fractional charges in the exact RCFT massless spectrum, with no argument why we find ourselves in such a special universe. Secondly it would undermine the usual assumption that the low-energy spectrum should be that of the MSSM, and nothing more. Other vector-like particles do not satisfy the same strict limits as stable, fractionally charged ones, and without having such limits, the logical conclusion would be to predict their discovery at the LHC, rather than bend

\footnotetext{
${ }^{3}$ Note however that even for fractionally charged exotics it is possible to find multiplets that have no impact on gauge coupling unification. An example is a standard model family with the opposite sign for all $Y$-charges, which gives rise to third-integral charges. Just like an extra family, this affects only the value of the unified coupling, but not the convergence itself.
} 
string theory out of shape to fit non-existent data.

We conclude that it is important to find out if exact heterotic string spectra without massless fractional charges are indeed rare, and to do so in as many different constructions as are accessible. We will discuss our results for heterotic weight lifting in section 4.

\section{Heterotic Weight Lifting}

The starting point of the construction of heterotic strings using interacting rational conformal field theory is a diagonal modular invariant partition function with identical building blocks in the bosonic and the fermionic sectors. In the fermionic sector the choice of building blocks is tightly constraint by the requirement of world-sheet supersymmetry. But on the bosonic side the only constraint is conformal invariance, and hence a much larger set of building blocks is in principle available. However modular invariance makes it nearly impossible to combine distinct building blocks in the left and right sectors.

Heterotic weight lifting [11] is a special case of a presumably very large set of modifications of the heterotic string, based on the idea of replacing some building blocks in the bosonic sector by isomorphic ones in the sense of the modular group. The difficulty is then to find building blocks that are isomorphic, but not identical, and have the same central charge. Some examples are known, such as the affine algebras of the $E_{8} \times E_{8}$ and $S O(32)$ heterotic strings or the meromorphic $c=24$ CFTs [23]. All of these are CFTs with just a single primary field. But what we need here are building blocks that are isomorphic to $N=2$ minimal models.

It is an interesting question whether there exist CFTs with the same $S$ and $T$ matrices and central charge as a given $N=2$ minimal model, without being identical to it. We do not know of any example. However, we can remove the superfluous $E_{8}$ factor that is present in the bosonic sector of a $(2,2)$ heterotic string and allow the central charge to differ by 8 . There is a well-known example of such an isomorphism: $S O(N)_{1} \times E_{8}$ versus $S O(N+16)_{1}$.

In a nutshell, the method works as follows. We start with the level-k minimal model times $E_{8}$. The minimal model can be realized as a coset CFT:

$$
\frac{S U(2)_{k} \times O(2)}{U_{2(k+2)}}
$$

The precise construction of this CFT involves "field identification", which can be viewed as a formal extension of the chiral algebra with a spin-0 current of order 2 . Now we "deconstruct" this coset construction by formally removing the field identification, then we embed the denominator algebra $U_{2(k+2)}$ in $E_{8}$ instead of $S U(2)_{k} \times O(2)$, and then we re-establish the field identification as a standard simple current extension.

The resulting CFT has a chiral algebra $S U(2)_{k} \times O(2) \times X_{7}$, where $X_{7}$ is the remainder of $E_{8}$ after dividing out $U_{2(k+2)}$. In other words, the tensor product $U_{2(k+2)} \times X_{7}$ can be chirally extended to $E_{8}$. The factor $X_{7}$ has central charge 7 , and its modular transformation matrices $S$ and $T$ are the complex conjugates of those of $U_{2(k+2)}$. In [11] 
a list of 33 such combinations was given, which was not claimed to be complete. We will call the isomorphic $N=0$ CFT the "lift" of the corresponding $N=2$ CFT. For some values of $k$ no such lift was found, and for some values there are two. Meanwhile we have attempted to find more examples, but so far without success. Nevertheless, we feel that the list published in [11] is just the "low-hanging fruit". These examples were easy to get, because they can be realized in terms of tensor products of affine Lie algebras. The presence of these affine Lie algebras is important in order to reproduce the large simple current groups of the minimal models, but there may well be other ways of achieving that.

Another possibility considered in [11] is to replace two minimal model factors and the $E_{8}$ factor by an isomorphic CFT. One example was found, but it will not be considered here.

A Gepner model is characterized by a set of values $\left(k_{0}, \ldots, k_{M}\right)$ for the levels of the minimal model factors. In principle each of the values can be lifted, provided a lift exists. We will denote a lifted Gepner model by $\left(k_{0}, \ldots, \widehat{k}_{i}, \ldots, k_{M}\right)$ if the $i^{\text {th }}$ factor is lifted. If $k_{i}$ has two distinct lifts, we use the notation $\tilde{k}_{i}$ for the second one. There are 168 standard Gepner models. Applying all 33 single lifts listed in [11], we end up with 435 lifted Gepner models.

Note that we may combine lifts with any modular invariant partition function of the standard Gepner models, including exceptional ones. Here we will not consider the latter. Apart from that, we will consider exactly the same set of MIPFs as in our previous paper [1, allowing in particular the breaking of $S O(10)$ to any of the eight subgroups listed there. We also allow breaking of world-sheet and space-time supersymmetry in the bosonic sector. Of course this is already the case as soon as we replace one of the minimal models by its lift. However, in addition to that we also allow the space-time supersymmetry current and the world-sheet supersymmetry alignment currents of the fermionic sector to be mapped to any isomorphic current in the bosonic sector.

\section{Fractional Charges and Group Types}

In [1] we presented a detailed discussion of $S O(10)$ breaking and the associated fractional charges (by which we always mean electric charges of color singlet particles). In the present work, $S O(10)$ breaking is treated in exactly the same way, and therefore a priori the possibilities are exactly the same. We just summarize the main points for convenience.

The heterotic string revolutionized string theory in 1984 [24] partly because it became clear almost immediately [25] that chiral four-dimensional spectra could be obtained with a remarkable resemblance to the observed particle spectrum. It is not an exaggeration to say that by merely imposing chirality and four uncompactified space-time dimensions one is almost inescapably led to spectra consisting of a number of families of (16)'s of $S O(10)$ (or in the special cases discovered earliest [25], (27)'s of $E_{6}$ ). This is especially easy to demonstrate by using the "bosonic string map" [26].

Unfortunately, this does not work so beautifully anymore if one tries to break $S O(10)$ to $S U(3) \times S U(2) \times U(1)$. This cannot be done with a field-theoretic Higgs mechanism, be- 
cause the required Higgs representations cannot occur as massless states for affine $S O(10)$ at level 1. Instead one can do the breaking directly in string theory. In our language, the breaking amounts to writing the theory in terms of $S U(3) \times S U(2) \times U(1)_{30}$ affine Lie algebras and not allowing the $S U(5)$ roots to appear in the massless spectrum. Here $U(1)_{2 N}$ denotes a free boson compactified on a circle such that there are $N$ primary fields with conformal weights $q^{2} / 4 N$, for $q=-N+1, \ldots, N$. Applying the standard group theory breaking $S U(5) \supset S U(3) \times S U(2) \times U(1)$ to affine $S U(5)$ level 1 yields $S U(3)_{1} \times S U(2)_{1} \times U(1)_{30}$ (we will omit the level indices on $S U(3)$ and $S U(2)$ henceforth). However, the only way to eliminate the $S U(5)$ roots while respecting modular invariance is by allowing some of the fractionally charged representations to appear. They must appear in the full theory, although not necessarily in the massless sector. In other contexts (orbifolds, Calabi-Yau constructions) the breaking is often achieved by using background gauge fields on Wilson lines, but no matter how it is done, as long as the result can be described in terms of $S U(3) \times S U(2) \times U(1)_{30}$ as above, this description applies.

Rather than breaking $S O(10)$ we start from its sub-algebra $S U(3) \times S U(2) \times U(1)_{30} \times$ $U(1)_{20}$, which we extend by a simple current of order 30 to $S O(10)$ in the fermionic sector. In the bosonic sector we allow some powers of this current to be replaced by other currents of the same order and relative monodromies. This allows in total eight different subgroups of $S O(10)$, listed in Table 1 . Which of these groups can be realized for a given tensor product depends on the values of the levels $k_{i}$ exactly as described in [1], and independent of the lifting. Each of these groups can still be further extended by means of currents with components outside $S O(10)$.

In this setup the first three factors, $S U(3) \times S U(2) \times U(1)_{30}$, are destined to become the standard model gauge group. Only a limited set of representations of this group has conformal weight less than or equal to one, and can occur in the massless spectrum. These are the standard quark and lepton multiplets Q, U, D, L, E and their conjugates, singlets and the $S U(3) \times S U(2) \times U(1)$ representations $\left(3,2, \pm \frac{5}{6}\right)$. The latter have weight one, and occur as extended roots in $S U(5)$ GUT models (yielding the familiar $X$ and $Y$ bosons). These representations do not occur as matter particles. All other representations that can occur in the massless spectrum violate the standard model charge integrality sum rule $\frac{t}{3}+\frac{s}{2}+Y \in \mathbf{Z}$ (where $t$ is $S U(3)$ triality and $s$ is the $S U(2)$ spin, modulo 2) and hence lead to color singlets with fractional electric charge. In the following we reserve the name "exotics" to particles that violate the charge integrality sum rule.

The $S U(3) \times S U(2) \times U(1)$ embedding we consider here is the standard one which would be obtained if one breaks $S O(10)$ to $S U(5)$ and then to $S U(3) \times S U(2) \times U(1)_{Y}$, with $U(1)_{Y}$ completely embedded in $S U(5)$. It is singled out by the requirement that the couplings converge in the canonical $S U(5)$ way. This embedding offers the best opportunity to understand the two "GUT miracles" (coupling convergence and family structure) in a natural way in heterotic strings, and perhaps even in string theory in general. It follows from the structure of the CFT that in this context the only fractional electric charges that can occur are $\frac{1}{2}, \frac{1}{3}$ and $\frac{1}{6}$. There are many other ways of embedding the standard model in the heterotic string. Examples are flipped $S U(5)$ models, which provide a different way to embed the standard model in $S O(10)$. Furthermore, in $(2,2)$ models one may consider 
general embeddings of $U(1)_{Y}$ in $E_{6}$, as was done for example in [6] and [7]. In these papers other fractional charges, like $\frac{1}{5}$, are mentioned. This must be due to a different choice for the $U(1)$ embedding. Furthermore one may consider affine Lie algebras $S U(3)$ and $S U(2)$ at several higher levels, and distribute them freely over the available $c=22$ internal CFT. In the rest of this paper we will often use the term "heterotic strings" to refer to the specific $S U(3) \times S U(2) \times U(1)_{30}$ class described above.

\subsection{Group Types}

If the algebra $S U(3) \times S U(2) \times U(1)_{30}$ is extended, the set of allowed fractional charges can be reduced, but it can only be reduced to integers if the extension includes $S U(5)$. For each group type, the allowed fractional charges are listed in the last two columns of Table 1. The first of these columns lists the fractional charges one would expect on the basis of the gauge group (given the fact that by construction the quantization of the $U(1)$ factor is in units of $\frac{1}{6}$ ), and the second column lists the true quantization in string theory, as explained in [1].

Of these eight groups, the $S U(5)$ GUT and the $S O(10)$ GUT are unacceptable, since, as explained above, they come without Higgs bosons to break the GUT gauge symmetry (we do not consider the possibility that such Higgses are generated dynamically as bound states of other massless matter). Higgs bosons to break the Pati-Salam group or the left-right symmetric groups to the standard model can exist in the massless spectrum, and hence these options will be considered viable. The most attractive possibility is nr. 2 , called "SM, $\mathrm{Q}=1 / 2$ ", because it has a gauge group closest to the standard model, and a charge quantum closest to 1 . Within the $S O(10)$ sector of the theory it has a gauge group which is exactly $S U(3) \times S U(2) \times U(1)$, times a superfluous extra $U(1)$. In some cases this extra $U(1)$ is B-L, but there are other possibilities.

In the figures we will distinguish these group types by means of the color codes $4^{4}$ shown in Fig. 1. In the text we will usually refer to them by the names in the second column of Table 1 .

\subsection{Anomalies}

In heterotic strings of the type considered here, anomalies cancel by means of the standard Green-Schwarz mechanism involving the $B_{\mu \nu}$ field. Note that string theories obtained by heterotic weight lifting do not have a known geometric interpretation, and cannot be derived in any known way by compactifying ten-dimensional heterotic strings. Hence the only way to derive anomaly factorization here is by computing the "elliptic genus" of the CFT, and using its modular properties, as explained in [27. This computation applies in this case, because it is valid in complete generality for all $(0,2)$ CFT constructions (in fact even for $(0,1)$ constructions).

\footnotetext{
${ }^{4}$ The color codes used here are slightly different than those used in [1] in order to make them more easily distinguishable in back and white printouts.
} 


\begin{tabular}{|c|c|c||c|c|}
\hline \hline Nr. & Name & Gauge group & Grp. & CFT \\
\hline 0 & $\mathrm{SM}, \mathrm{Q}=1 / 6$ & $S U(3) \times S U(2) \times U(1) \times U(1)$ & $\frac{1}{6}$ & $\frac{1}{6}$ \\
1 & $\mathrm{SM}, \mathrm{Q}=1 / 3$ & $S U(3) \times S U(2) \times U(1) \times U(1)$ & $\frac{1}{6}$ & $\frac{1}{3}$ \\
2 & $\mathrm{SM}, \mathrm{Q}=1 / 2$ & $S U(3) \times S U(2) \times U(1) \times U(1)$ & $\frac{1}{6}$ & $\frac{1}{2}$ \\
3 & $\mathrm{LR}, \mathrm{Q}=1 / 6$ & $S U(3) \times S U(2)_{L} \times S U(2)_{R} \times U(1)$ & $\frac{1}{6}$ & $\frac{1}{6}$ \\
4 & $\mathrm{SU}(5) \mathrm{GUT}$ & $S U(5) \times U(1)$ & 1 & 1 \\
5 & $\mathrm{LR}, \mathrm{Q}=1 / 3$ & $S U(3) \times S U(2)_{L} \times S U(2)_{R} \times U(1)$ & $\frac{1}{6}$ & $\frac{1}{3}$ \\
6 & Pati-Salam & $S U(4) \times S U(2)_{L} \times S U(2)_{R}$ & $\frac{1}{2}$ & $\frac{1}{2}$ \\
7 & $\mathrm{SO}(10) \mathrm{GUT}$ & $S O(10)$ & 1 & 1 \\
\hline
\end{tabular}

Table 1: List of all standard model extensions within $S O(10)$ and the resulting group theory and CFT charge quantization (last two columns). We refer to these subgroups either by the label in column 1 or by the name in column 2, where "LR" stands for left-right symmetric.

This result implies that the anomaly factorizes in the standard way in a factor linear in $F$ and $R$, times a factor $\operatorname{Tr} F^{2}-\operatorname{Tr} R^{2}$, where $F$ is evaluated in the vector representation of an orthogonal group. Furthermore it follows that $\operatorname{Tr} F^{2}$ receives contributions from every factor in the gauge group. If the $E_{8}$ factor in the gauge group is unbroken, it cannot produce a term of the form $\operatorname{Tr} F^{2} \operatorname{Tr} F^{\prime}$, where $F$ is an $E_{8}$ two-form and $F^{\prime}$ a $U(1)$ two-form. This is because the only non-trivial representation of $E_{8}$ level 1 that can be massless is the (248), and since this exactly saturates the conformal weight, there is no room for a $U(1)$ charge. Hence there are no massless representations charged under both $E_{8}$ and any $U(1)$. If $E_{8}$ cannot contribute to $\operatorname{Tr} F^{2}$, it follows that there cannot be a $\operatorname{Tr} F^{2}-\operatorname{Tr} R^{2}$ factor at all, and hence the anomaly must be identically 0 . Since heterotic weight lifting breaks the $E_{8}$ factor, it is no longer present in the gauge group. Therefore in general there can be a non-trivial Green-Schwarz mechanism in these models. This also implies that $U(1)$ factors can be anomalous. This may happen in particular to the standard model $U(1)$ factor $Y$ or the $U(1)$ corresponding to B-L. The corresponding $U(1)$ gauge boson will then become massive because of the Stueckelberg mechanism, absorbing the $B_{\mu \nu}$ field. Note that for this to happen, the $U(1)$ factor must really be anomalous. This is different from orientifold models: there even a non-anomalous $U(1)$ can acquire a mass from axion mixing. It follows that in a heterotic model with a certain number of standard model families, but no chiral exotics, $U(1)_{Y}$ must remain unbroken, because it is anomaly free. However, the extra $U(1)$ that commutes with the standard model gauge group within $S O(10)$ can be anomalous. This is because we are not requiring this charge to coincide with B-L for all quarks and leptons.

In the absence of fractionally charged matter, anomaly cancellation would force the 


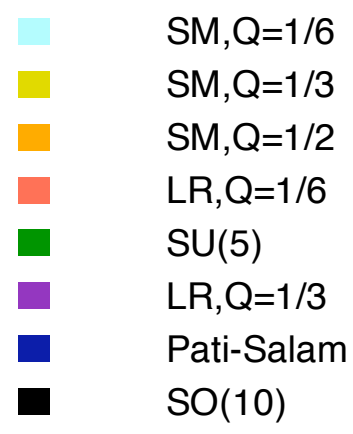

Figure 1: Color codes for group types.

spectrum into a certain number of standard model families. One question we will be interested in is to which extent the existence of fractionally charged matter changes that.

\subsection{Group type distributions}

First we will examine how often we find each group type if we simply select MIPFs at random. MIPF selection requires choosing a subgroup of the full set of simple currents, plus a discrete torsion matrix defined on that subgroup [28]. We have randomized this choice by first choosing $N$ random elements of the full simple current group, with equal weight for each element. Then we compute the subgroup generated by these $N$ elements. Finally we either enumerate all allowed discrete torsions defined on that subgroup if there are fewer than a hundred, or otherwise we take a random sample. The amount of time needed to compute spectra increases rapidly with $N$, and in practice values of $N$ larger than 4 are difficult to deal with. Our data are based on nearly saturated samples for $N=0,1$ and 2, but on incomplete random samples for $\mathrm{N}=3$ and 4 . Obviously the precise results depend on various details of the sampling method, and in particular on the number of MIPF samples taken for each $N$, but we have checked that the gross features are not strongly dependent on those

In principle there is an exact correspondence between MIPFs of standard and lifted Gepner models: the same MIPF gives rise to the same group type. Hence if one randomly select tensor products and MIPFs one would get the same distribution for these different group types (though the fact that different standard Gepner models may have different numbers of lifts, as well as differences in randomization introduce some discrepancies). This distribution is shown for the lifted Gepner models in Fig. 2, It is based on $9.4 \times 10^{8}$ MIPFs, each counted once, before comparing spectra and identifying identical ones.

This distribution contains all spectra, including those with chiral fractionally charged particles. If we require absence of chiral exotics, the distribution is the one shown in Fig. 3. This figure is based on $6.8 \times 10^{8}$ MIPFs. Note that requiring absence of chiral exotics reduces the number of spectra by only about $30 \%$. The reduction is strongest for the spectra with third-integer charges and surprisingly small for those with sixth-integer 


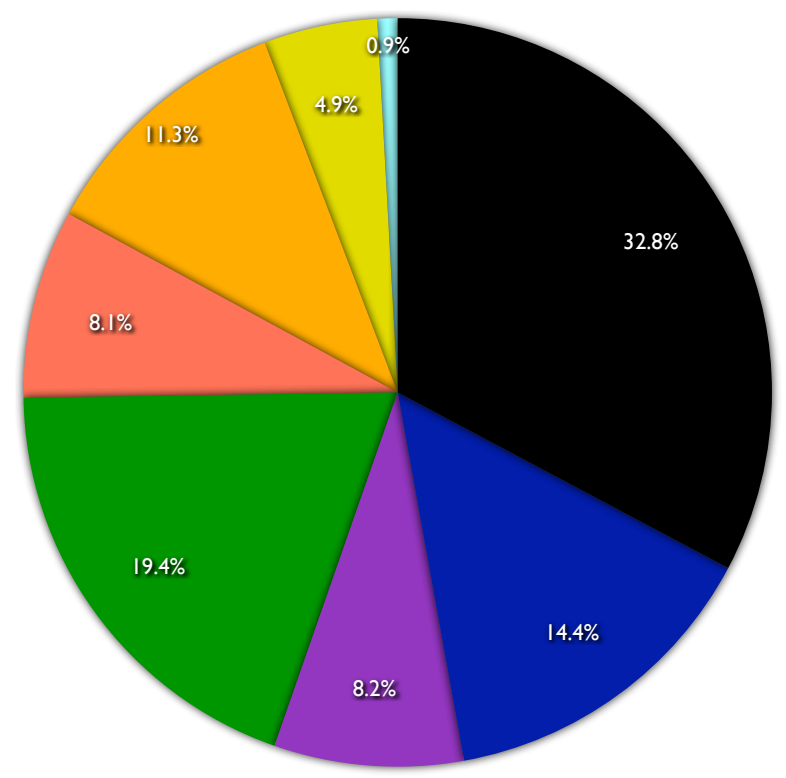

Figure 2: Overall distribution of group types.

charges. The two half-integer charged types, Pati-Salam and SM, $Q=\frac{1}{2}$ behave rather differently: the former is only reduced by less than $10 \%$, whereas the latter is reduced by a factor 10. In the vast majority of these spectra, fractionally charged exotics are present in the massless spectrum, but they are vector-like.

In most cases, the absence of chiral exotics is simply due to the fact that the entire spectrum is non-chiral. So as a final step we consider only those cases that have at least one chiral family. Then we get Fig. 4. Now there are $1.8 \times 10^{8}$ spectra left. The $Q=\frac{1}{6}$ spectra are reduced to just a few hundred, and are not visible. The $Q=\frac{1}{3}$ spectra nrs. 1 and 5 occur respectively about 100.000 and 24.000 times, and are also invisible. The only ones that remain with a substantial frequency are Pati-Salam, SM, $Q=\frac{1}{2}$, and the two GUT models $S U(5)$ and $S O(10)$. Interestingly, the $S O(10)$ spectra are reduced much more than the $S U(5)$ models, i.e. the latter are more often chiral.

\subsection{Fractional charges}

Often the appearance of fractionally charged particles in broken heterotic GUTs is handwaved away. Indeed, they may be massive or confined by additional forces, but the elegance of the original GUTs $(S U(5)$ or $S O(10))$ is lost in either case. More seriously, we loose a deeper understanding of why the standard model matter comes in the form of the chiral families we observe. Without the availability of fractionally charged representations, anomaly cancellation necessarily imposes the observed family structure on the chiral spectrum. If in addition one makes the plausible assumption that only the chiral spectrum survives at low energies, the observed family structure is nicely explained. This 


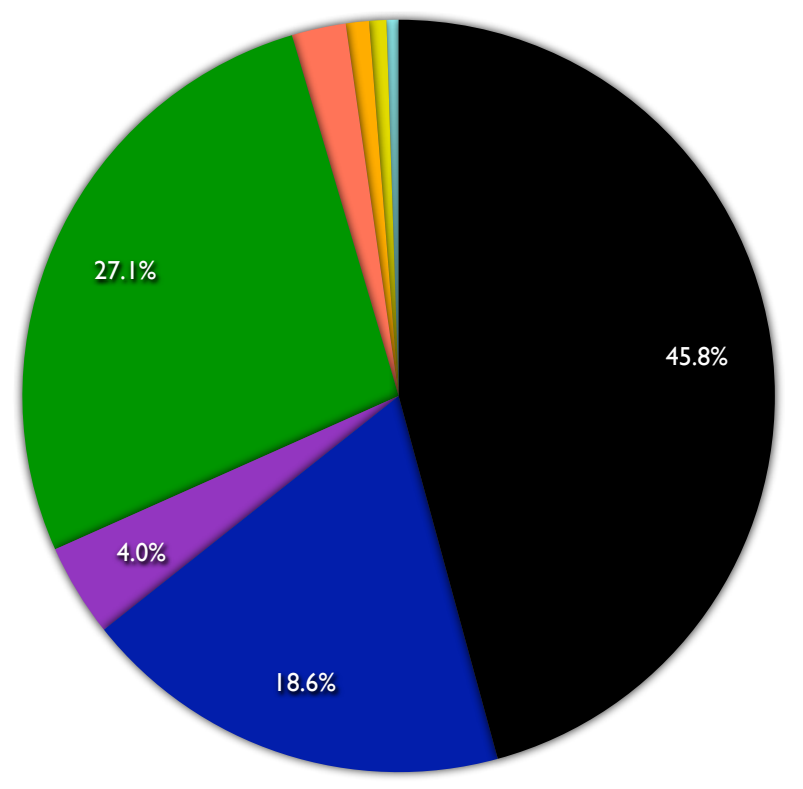

Figure 3: Overall distribution of group types for spectra without chiral exotics.

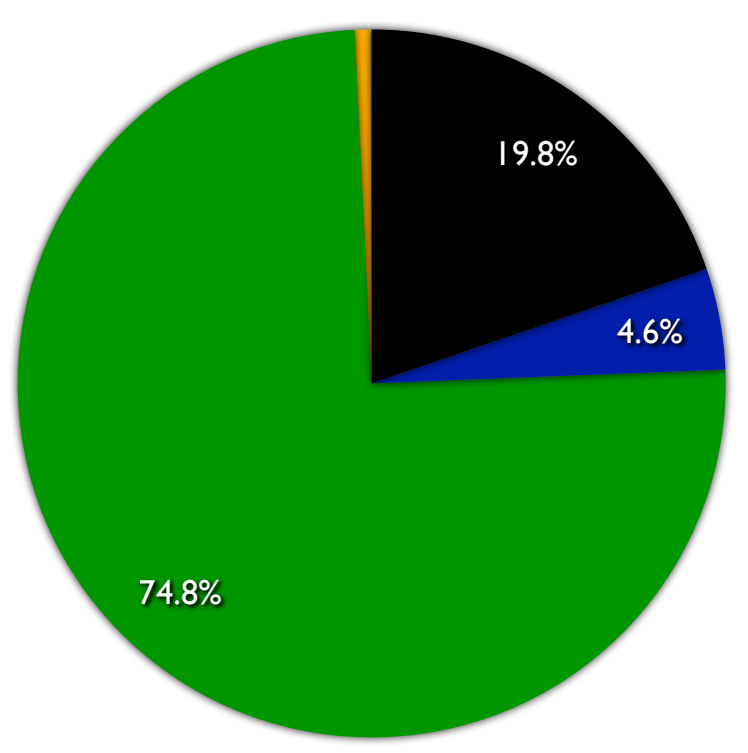

Figure 4: Overall distribution of group types without chiral exotics and at least one family. 
is indeed true in $S U(5)$ field theory models, but not in heterotic GUTs of the type considered here (it would be true in heterotic GUTs based on higher level affine Lie algebras, but in that case the set of allowed massless representations is not naturally limited to the observed ones). As soon as fractionally charged matter is available in principle, there are several other solutions to anomaly cancellation. Therefore the observed chiral structure of a standard model family is not understood in this class of heterotic strings, even though it seemed that with (16)'s of $S O(10)$ we were on the right track. If the initial emergence of (16)'s of $S O(10)$ is considered a success, by the same standards the appearance of fractionally charged particles must be seen as a failure.

It is well-known [29] that in models of this type (heterotic strings with $S O(10)$ broken to $S U(3) \times S U(2) \times U(1))$ quarks and leptons are not really unified into a single (16) of $S O(10)$. They typically originate from several different (16)'s in the original manifold. However, if indeed there were no fractional charges available, one could legitimately argue that in this class of heterotic strings the group-theoretical fact that a family of quarks and leptons fits in a (16) is understood, even if they do not really belong to the same multiplet. As soon as fractionally charged anomaly-free multiplets are available in principle, the best one can still hope for is that models with the observed family structure dominate the landscape statistically. Observe that in field-theoretical $S O(10)$ models, upon breaking $S O(10)$, a representation (16) automatically decomposes into standard quarks and leptons, whereas in string-theoretic $S O(10)$ models in the heterotic class considered here, this is not automatic. Of course one can impose the bias that only standard families are allowed in the chiral spectrum, but then on cannot argue anymore that string theory fully explains the family structure.

\subsubsection{Chiral fractional charges}

In RCFT constructions like the one we are discussing here, $S U(3) \times S U(2) \times U(1)_{30}$ spectra are sampled without any further bias towards the standard model family structure (of course the gauge group choice itself is a bias). So in this context it becomes natural to ask if, despite the existence of other options, the standard model family structure still dominates in a statistical sense.

The "other options" are models with chiral multiplets that are not of the form of a standard model family. We merely count these spectra before rejecting them, in order to see to what extent one can still argue that the structure of a standard model family is understood in this class of heterotic strings.

As remarked above, about $71 \%$ of randomly generated spectra turned out to be free of chiral exotics, whereas for the standard Gepner models this ratio is about $63 \%$. One might be tempted to conclude that fractional charges are generically vector-like in these models. But this is a slightly misleading statement for two reasons: on the one hand the set is dominated by $S O(10)$ and $S U(5)$ models, for which this is automatically true, and on the other hand it is dominated by non-chiral spectra, for which it is also automatically true. Another way to ask the question is: which percentage of the chiral spectra with a broken GUT group (i.e. all types except $S U(5)$ and $S O(10)$ ) and a non-anomalous $Y$-charge have 
a standard family structure with at least one family? For standard Gepner models, the answer is about 20\%. For lifted Gepner models it is about 4.6\%. These numbers are not extremely small, but it is hard to argue that the observed family structure is understood in this class of models. Anthropic arguments can be expected to enlarge these ratios (note that we are discussing chiral, and hence necessarily light or even exactly massless particles here). Indeed, we know already that for suitable parameter values the standard family structure can lead to the complex nuclear physics and chemistry needed for life, but this is not likely to be the case for the entire set of exotic chiral representations of $S U(3) \times S U(2) \times U(1)$ that emerges from these heterotic strings. Indeed, some of them are lacking some or all standard quarks and leptons. However, analyzing this is unfortunately far beyond anyone's capabilities.

It would obviously be very interesting to have information about this issue in other constructions (such as orbifolds, Calabi-Yau compactifications) but usually spectra with chiral exotics are not even considered in these approaches. In [30, 31, 32] however, a similar analysis has been done for Pati-Salam models based on free fermions. Their conclusions appear to agree with ours qualitatively: spectra with chiral exotics dominate those with only standard families by about a factor of five.

\subsubsection{Vector-like fractional charges}

In most of the aforementioned $4.6 \%$ of chiral models without chiral exotics, the fractionally charged exotics exist as vector-like particles. Here we mean vector-like with respect to $S U(3) \times S U(2) \times U(1)$. We did not examine chirality with respect to other factors in the gauge group. Spectra of this kind are potentially acceptable, provided they acquire a very large mass in a proper string vacuum, as discussed in section 2 .

\subsubsection{Massive fractional charges}

We also found examples where the fractional charges are completely absent from the massless spectrum. In [1] we found only very few examples, all of them with zero families; for lifted Gepner models we also have examples with a non-vanishing, though always even $(2,4,6,8,12$ or 24) number of families. These were found only for the Pati-Salam and $\mathrm{SM}, \mathrm{Q}=\frac{1}{2}$ group types; in other words, only if the CFT charge quantum is $\frac{1}{2}$. They occur very rarely indeed. To get an idea, let us see the precise numbers for the most common one, the Pati-Salam model. In total, we found about 864.000 distinct ${ }^{5}$ PatiSalam type models without chiral exotics, of which about 100.000 were chiral, i.e. they have a non-vanishing number of standard model families. About 6000 Pati-Salam models had no massless exotics at all, but of these 6000 only 143 had a non-zero number of chiral families. For type SM, $\mathrm{Q}=\frac{1}{2}$ these numbers are respectively 75.000, 36.000, 330 and 36 . In both cases, the chance that a chiral model has no fractionally charged exotics at all

\footnotetext{
${ }^{5}$ Note that here each distinct spectrum is counted only once, whereas the foregoing figures were based on total occurrences
} 
is about 1 in 1000. It seems unreasonable to claim that this is the reason we observe no fractional charges experimentally.

This same issue has been investigated recently for free fermionic constructions in [30, 31, 32]. These authors only constructed Pati-Salam models and found a somewhat smaller rate for the absence of fractional charges, about $10^{-5}$, but did find examples with three families. This demonstrates that there does not exist a no-go theorem against such spectra in the general class of $(0,2)$ models. Given the fact that the total number of examples without massless fractional charges we found is of order 100, there is a chance that three family examples occur in our set as well at a rate of $10^{-5}$, but are still hidden in the noise.

The most important conclusion here is that non-exotic models are rare. As explained before, in our opinion in RCFT one should aim for absence of chiral exotics, but requiring complete absence of vector-like exotics (fractionally charged or not) is simply too much to ask for. To put it differently: if one were to reject models because they have some vector-like exotics at the level of exact RCFT, one risks loosing many examples that would survive perfectly if one perturbs the theory.

It is known that massless fractional charges can be avoided using Wilson line symmetry breaking in combination with freely acting discrete symmetries [6, 7]; for recent examples see e.g. [33, 34]. Let us emphasize that this is not in disagreement with the statement above. The question we are trying to answer is how common such non-exotic models are in the full class of examples that have chiral families. This question is not answered by focusing only on the case where massless fractional charges are absent by construction. It should be noted that most models in the "heterotic mini landscape" studied prior to [34] (see e.g. 20]) did have exact spectra with massless fractional charges, whose masses could be lifted by certain terms in the superpotential. It would be tremendous progress if a non-phenomenological argument could be found to explain why freely acting discrete symmetries should be favored over other ways to get the standard model from the heterotic string.

Nevertheless, it would be interesting to find out if the examples we found, or those of [30], can be understood in terms of some freely acting discrete symmetry (which is not manifest in our formalism), or if they are merely statistical fluctuations. The distribution shown in Fig. 13, and which will be discussed in section 6, suggests the latter, since the small peak at zero is not inconsistent with the rest of the distribution (for comparison, the peaks at zero in the first two plots of Fig. 16, to be discussed later, are clearly not statistical fluctuations).

\section{The Number of Families}

For comparison we show in Figs. 5 and 6 the distribution of the number of families for standard Gepner models (168 minimal model combinations, plus 59 with exceptional invariants of $S U(2)$ ) and lifted Gepner models (435 combinations). On the vertical axis we show the number of MIPFs. We distinguish MIPFs on the basis of the numbers of standard 
model representations Q, U, D, L, E and their conjugates, the total number of standard model singlets, the total number of fractionally charged particles (not counting their color or weak isospin degrees of freedom, but counting their dimension in any group that is not part of the standard model), the total number of gauge bosons, the observable part of the gauge group (gauge bosons that are neutral with respect to $S U(3) \times S U(2) \times U(1)$ are merely counted, but their mutual interactions are ignored), and any deviations from the expected charge quantization in the massless spectrum (for example, if on the basis of the CFT particles of color singlet charge $\frac{1}{6}$ are allowed, but in the observed spectrum these particles all turn out be massive).

Of course this is not strictly the same as distinguishing MIPFs, since two distinct MIPFs might still produce the same spectrum, either by coincidence, or by symmetryrelated degeneracies (for example the interchange of identical factors in the tensor product). If there are such degeneracies, it would be preferable to remove them anyway, so no harm is done by identifying such spectra. Furthermore, such symmetry-related spectra will be identical in all other respects as well. This still leaves the possibility of coincidental matches of only the massless spectrum. We have reasons to believe these are rare (more about this later), so that what we are plotting in Figs. 5 and 6 is indeed very close to the number of truly distinct MIPFs.

Obviously these plots are not true landscape distributions, i.e. distributions of nonsuper-symmetric moduli-stabilized string vacua. Furthermore one may question the relative weights for different MIPFs, and especially the relative weights given to different tensor products. However, the main point we want to stress in Fig. 5 is that in standard Gepner models the number three is hard to get, and in particular very much disfavoured in comparison to two and four, confirming the experience of two decades ago. This result remains true regardless of the measure one chooses, unless one can find an argument explaining why the $\left(1,16^{*}, 16^{*}, 16^{*}\right)$ (giving rise to the only 3 -family models in this set) should be enhanced by a huge factor over anything else.

The main result of the present paper is shown in Fig. 6. We observe that in the lifted Gepner model landscape an approximately exponential family distribution is obtained, where the number three is not especially disfavoured with respect to two and is even favoured with respect to four. Indeed, the impression one gets from this plot is that families with a factor of two and/or three are enhanced with respect to the average. Note for example the peaks at 6,9 and 12 families. Also for these models the same caveats regarding true landscape distributions apply. However in this case three family spectra do not just come from a single tensor product, but from 260 of the 435 combinations, so the issue of their relative weight is less important. Of course it is likely that moduli stabilization and supersymmetry breaking affect the shape of this distribution, but we are not aware of any effect that would single out the number three, either by means of a dip or a peak. It is much more plausible that such effects might change the slope, and indeed it is still possible that this suppresses three families with respect to two by a huge amount, but the optimistic interpretation of our results is that in the heterotic landscape, as soon as one moves away from the $(2,2)$ models, the number three is not especially difficult to obtain. 
Further evidence for the special role of the numbers two and three comes from studying the greatest common divisor (denoted $\Delta$ ) of the number of families for all the MIPFs of each lifted $N=2$ tensor product. The results are listed in Table 2, Most lifted Gepner models have a value $\Delta=1$, but $0,2,3,4$, and 6 also occur. By contrast, for standard Gepner models, the values of $\Delta$ we found were $0,2,3,6$ and 12 , with 1 completely absent and 3 occurring just once. The most noticeable difference is the large number of cases we get now with $\Delta=1$. There are no cases with $\Delta=5$ or any number larger than 6 . We do get spectra with 5,7 , or $11, \ldots$ families from the $\Delta=1$ models; the first number that we did not encounter at all is 31 .

The distribution of families was also studied recently in the context of heterotic strings using free fermions [32. These authors also find a roughly exponential fall-off with the number of families, but with a steeper slope of about a factor three per family. Even though in that case three is more suppressed, one could still conclude that three family spectra are not especially rare. An interesting difference with our results is the complete absence of spectra with seven families (or any larger odd number). This must perhaps be viewed as an artifact of free fermionic constructions.

\begin{tabular}{|c||c|}
\hline \hline$\Delta$ & Number \\
\hline 0 & 94 \\
1 & 199 \\
2 & 56 \\
3 & 61 \\
4 & 8 \\
5 & 0 \\
6 & 17 \\
\hline
\end{tabular}

Table 2: The number of lifted Gepner models with a given family quantum $\Delta$.

Our conclusion is quite different from what was observed for orientifolds in [4] and [5]. In this case the number of families drops off much faster, and there is a clear additional suppression of odd numbers with respect to even ones, which unfortunately is not understood. Consequently, three is suppressed with respect to two and four by two to three orders of magnitude. It is impossible to tell whether the heterotic results or the orientifolds results are more typical for the string landscape as a whole, but at least there is a better chance now that the difficulties with obtaining three families can be attributed to artifacts of the chosen method.

Note that Fig. 6 is dominated by just four group types, the phenomenologically useless (because unbroken) GUT groups $S U(5)$ and $S O(10)$, the Pati-Salam model, and 
the unextended standard model with charge quantization in half-integer units. The other four types are also present, as was already discussed in the previous section, but there are too few of them to be visible. To make them appear we can add the data for zero family models. This is shown for standard Gepner models in Fig. 7 and for lifted Gepner models in Fig. 8. Especially in the latter case this yields a huge bar at zero that dominates everything. Universes with zero families thus seem to dominate the ensemble, but this is not a problem, since they are anthropically unacceptable.

In comparison with the results of [32] we get a much larger peak at zero families. This can be explained, at least partly, as follows. Our plots are in terms of the group types introduced in the previous section. These group types define the subgroup of $S O(10)$ that is realized, but we do not put any constraints on the extension of these groups into the internal sector of the theory, whereas the authors of [32] require that there be no extension beyond the group they consider, the Pati-Salam model. Some of those extensions will render the gauge group non-chiral, and therefore can only contribute to the zero-family peak in the distribution, producing an abnormal enhancement of that peak.

Note that in Figs. 5 ...8 8 on the vertical axis we have plotted the number of distinct MIPFs, and not the absolute occurrence frequency. This explains why the relative distribution of group types appears different than in the pie charts shown in the previous section. If MIPFs are selected at random, the unbroken $S U(5)$ and $S O(10)$ cases tend to come out far more often, and hence they dominate the set. The reason for using total occurrence frequency rather than the total number of distinct MIPFs in the foregoing section was explained in [1: we do not distinguish MIPFs for spectra with chiral exotics.

In Table 3 we show all lifted Gepner models with a non-vanishing value for $\Delta$. In the first column the combination of levels is shown, with a hat indicating which factor is lifted. For some values of the level $k$ two lifts are possible. The second one is indicated by a tilde instead of a hat. Column 2 shows the value of $\Delta$ and column 3 the maximal number of families for that model. Column 4 lists the number of distinct 3-family spectra we obtained, and column 5 lists the total number of distinct $N$ family spectra, with $N \geq 1$. In both of these columns we have modded out mirror symmetry, i.e. a spectrum and its mirror pair is just counted once.

As explained in [1] the full set of spectra we obtain is mirror symmetric. This is a statement about RCFT, and does not necessarily imply anything about an underlying geometry. Typically, in order to encounter a mirror partner of a given spectrum it is necessary to add one simple current twist. Therefore, if we go to arbitrary numbers of generators of the simple current subgroup, we will eventually find all mirror pairs. For practical reasons, we have limited this number to 4 . By checking if the set of spectra is closed under mirror symmetry we can get a rough idea about the completeness of the set. For this reason we have indicated in the last column which percentage of the total sets of MIPFs is lacking a mirror partner. As expected, this number tends to be largest for tensor combinations with many factors, because here the limitation to four generators is most restrictive. 
Table 3: Results for lifted Gepner models

\begin{tabular}{|c||c|c|c|c|c|}
\hline model & $\Delta$ & Max. & 3 family & $N$ fam. $(N>0)$ & Missing Mirrors \\
\hline$(\widehat{1}, 10,11,154)$ & 6 & 6 & 0 & 4 & $0.00 \%$ \\
$(1,10, \widehat{1}, 154)$ & 6 & 6 & 0 & 8 & $0.00 \%$ \\
$(1,10, \widehat{12}, 82)$ & 1 & 8 & 10 & 69 & $0.00 \%$ \\
$(\widehat{1}, 10,13,58)$ & 1 & 18 & 189 & 563 & $0.71 \%$ \\
$(1,10, \widehat{13}, 58)$ & 1 & 64 & 11 & 295 & $0.34 \%$ \\
$(1,10,13, \widehat{58})$ & 1 & 42 & 7 & 103 & $0.00 \%$ \\
$(\widehat{1}, 10,14,46)$ & 3 & 12 & 126 & 172 & $8.14 \%$ \\
$(1,10, \widehat{14}, 46)$ & 1 & 14 & 96 & 648 & $12.50 \%$ \\
$(\widehat{1}, 10,18,28)$ & 1 & 17 & 126 & 560 & $6.79 \%$ \\
$(\widehat{1}, 10,19,26)$ & 3 & 6 & 8 & 12 & $0.00 \%$ \\
$(1,10, \widehat{19}, 26)$ & 3 & 15 & 2 & 10 & $0.00 \%$ \\
$(1,10,19, \widehat{26})$ & 1 & 12 & 1 & 9 & $0.00 \%$ \\
$(\widehat{1}, 10,22,22)$ & 3 & 24 & 117 & 215 & $24.19 \%$ \\
$(\widehat{1}, 1,1,1,10,10)$ & 3 & 30 & 757 & 1194 & $31.49 \%$ \\
$(\widehat{1}, 1,1,1,1,1,1,1,1)$ & 3 & 33 & 304 & 584 & $40.07 \%$ \\
$(1,1,1,1,1,1,1, \widehat{4})$ & 2 & 24 & 0 & 437 & $33.64 \%$ \\
$(\widehat{1}, 1,1,1,1,1,1,4)$ & 3 & 33 & 359 & 670 & $27.76 \%$ \\
$(1,1,1,1,1, \widehat{2}, 10)$ & 1 & 24 & 26 & 226 & $15.04 \%$ \\
$(\widehat{1}, 1,1,1,1,2,10)$ & 3 & 30 & 98 & 343 & $26.24 \%$ \\
$(1,1,1,1,1, \widehat{4}, 4)$ & 1 & 24 & 344 & 2986 & $37.51 \%$ \\
$(\widehat{1}, 1,1,1,1,4,4)$ & 3 & 33 & 1827 & 3054 & $27.96 \%$ \\
$(\widehat{1}, 11,11,76)$ & 6 & 6 & 0 & 2 & $0.00 \%$ \\
$(1, \widehat{11}, 11,76)$ & 3 & 6 & 4 & 7 & $0.00 \%$ \\
$(1,11, \widehat{11}, 76)$ & 2 & 8 & 0 & 6 & $0.00 \%$ \\
$(1,1,1,1,2,2, \widehat{4})$ & 1 & 16 & 0 & 100 & $31.00 \%$ \\
$(\widehat{1}, 1,1,1,2,2,4)$ & 3 & 24 & 81 & 186 & $20.43 \%$ \\
$(1,1,1,1, \widehat{2}, 2,4)$ & 1 & 24 & 13 & 175 & $29.14 \%$ \\
$(\widehat{1}, 1,1,1,5,40)$ & 3 & 18 & 12 & 25 & $36.00 \%$ \\
$(1,1,1,1, \widehat{5}, 40)$ & 2 & 8 & 0 & 21 & $23.81 \%$ \\
$(\widehat{1}, 1,1,1,6,22)$ & 3 & 24 & 64 & 143 & $14.69 \%$ \\
$(1,1,1,1, \widehat{6}, 22)$ & 1 & 16 & 0 & 81 & $13.58 \%$ \\
$(\widehat{1}, 1,1,1,7,16)$ & 3 & 27 & 303 & 523 & $29.45 \%$ \\
\hline & & & & & Continued on next page \\
\hline
\end{tabular}


Table 3 - continued from previous page

\begin{tabular}{|c||c|c|c|c|c|}
\hline model & $\Delta$ & Max. & 3 family & $N$ fam. $(N>0)$ & Missing Mirrors \\
\hline$(\widehat{1}, 1,1,1,8,13)$ & 3 & 18 & 7 & 37 & $8.11 \%$ \\
$(1,1,1,1, \widehat{8}, 13)$ & 2 & 16 & 0 & 19 & $0.00 \%$ \\
$(\widehat{1}, 1,1,2,4,10)$ & 3 & 30 & 706 & 1377 & $27.09 \%$ \\
$(1,1,1, \widehat{2}, 4,10)$ & 1 & 24 & 315 & 1542 & $25.42 \%$ \\
$(1,1,1,2, \widehat{4}, 10)$ & 1 & 24 & 114 & 1245 & $22.25 \%$ \\
$(\widehat{1}, 1,1,4,4,4)$ & 3 & 33 & 4117 & 6732 & $28.58 \%$ \\
$(1,1,1, \widehat{4}, 4,4)$ & 1 & 32 & 1044 & 9903 & $22.56 \%$ \\
$(1, \widehat{12}, 12,40)$ & 1 & 18 & 21 & 123 & $0.00 \%$ \\
$(\widehat{1}, 1,2,12,82)$ & 3 & 18 & 28 & 55 & $0.00 \%$ \\
$(1,1, \widehat{2}, 12,82)$ & 1 & 14 & 16 & 70 & $0.00 \%$ \\
$(1,1,2, \widehat{12}, 82)$ & 2 & 8 & 0 & 15 & $0.00 \%$ \\
$(\widehat{1}, 1,2,13,58)$ & 1 & 18 & 42 & 156 & $4.49 \%$ \\
$(1,1, \widehat{2}, 13,58)$ & 1 & 14 & 22 & 160 & $3.12 \%$ \\
$(1,1,2, \widehat{13}, 58)$ & 1 & 18 & 1 & 60 & $1.67 \%$ \\
$(1,1,2,13, \widehat{58})$ & 2 & 32 & 0 & 19 & $5.26 \%$ \\
$(\widehat{1}, 1,2,14,46)$ & 3 & 15 & 144 & 211 & $6.16 \%$ \\
$(1,1, \widehat{2}, 14,46)$ & 1 & 12 & 42 & 174 & $0.00 \%$ \\
$(1,1,2, \widehat{14}, 46)$ & 1 & 12 & 9 & 195 & $2.05 \%$ \\
$(\widehat{1}, 1,2,16,34)$ & 3 & 21 & 368 & 734 & $19.75 \%$ \\
$(1,1, \widehat{2}, 16,34)$ & 1 & 18 & 73 & 816 & $12.50 \%$ \\
$(\widehat{1}, 1,2,18,28)$ & 1 & 12 & 98 & 247 & $1.62 \%$ \\
$(1,1, \widehat{2}, 18,28)$ & 1 & 12 & 42 & 204 & $1.96 \%$ \\
$(\widehat{1}, 12,19,19)$ & 3 & 6 & 14 & 18 & $0.00 \%$ \\
$(1, \widehat{12}, 19,19)$ & 1 & 14 & 0 & 9 & $0.00 \%$ \\
$(1,12, \widehat{19}, 19)$ & 6 & 6 & 0 & 1 & $0.00 \%$ \\
$(\widehat{1}, 1,2,2,2,10)$ & 3 & 24 & 219 & 458 & $5.68 \%$ \\
$(1,1, \widehat{2}, 2,2,10)$ & 1 & 36 & 263 & 1963 & $21.40 \%$ \\
$(\widehat{1}, 1,2,22,22)$ & 3 & 30 & 200 & 481 & $13.31 \%$ \\
$(1,1, \widehat{2}, 22,22)$ & 1 & 24 & 43 & 471 & $11.46 \%$ \\
$(\widehat{1}, 1,2,2,4,4)$ & 3 & 24 & 285 & 771 & $13.62 \%$ \\
$(1,1, \widehat{2}, 2,4,4)$ & 1 & 24 & 24 & 854 & $17.80 \%$ \\
$(1,1,2,2, \widehat{4}, 4)$ & 1 & 32 & 81 & 1555 & $20.19 \%$ \\
$(\widehat{1}, 1,3,13,13)$ & 1 & 24 & 220 & 569 & $20.74 \%$ \\
\hline & & & & Continued on next page \\
\hline
\end{tabular}


Table 3 - continued from previous page

\begin{tabular}{|c||c|c|c|c|c|}
\hline model & $\Delta$ & Max. & 3 family & $N$ fam. $(N>0)$ & Missing Mirrors \\
\hline$(1,1, \widehat{3}, 13,13)$ & 1 & 48 & 28 & 580 & $5.52 \%$ \\
$(1,1,3, \widehat{13}, 13)$ & 1 & 36 & 47 & 475 & $6.53 \%$ \\
$(\widehat{1}, 13,13,28)$ & 1 & 34 & 335 & 1093 & $5.67 \%$ \\
$(1, \widehat{13}, 13,28)$ & 1 & 80 & 145 & 1184 & $2.20 \%$ \\
$(\widehat{1}, 13,18,18)$ & 1 & 24 & 37 & 240 & $0.00 \%$ \\
$(1, \widehat{13}, 18,18)$ & 1 & 48 & 10 & 137 & $0.00 \%$ \\
$(\widehat{1}, 1,3,7,43)$ & 1 & 18 & 51 & 94 & $9.57 \%$ \\
$(1,1, \widehat{3}, 7,43)$ & 1 & 24 & 6 & 114 & $8.77 \%$ \\
$(\widehat{1}, 1,3,8,28)$ & 1 & 22 & 251 & 922 & $7.70 \%$ \\
$(1,1, \widehat{3}, 8,28)$ & 1 & 48 & 145 & 1279 & $15.72 \%$ \\
$(1,1,3, \widehat{8}, 28)$ & 1 & 32 & 42 & 334 & $4.49 \%$ \\
$(\widehat{1}, 1,4,10,10)$ & 3 & 30 & 2203 & 3775 & $11.42 \%$ \\
$(1,1, \widehat{4}, 10,10)$ & 1 & 24 & 269 & 2842 & $36.17 \%$ \\
$(\widehat{1}, 14,14,22)$ & 3 & 24 & 34 & 79 & $3.80 \%$ \\
$(1, \widehat{1}, 14,22)$ & 1 & 12 & 53 & 379 & $15.57 \%$ \\
$(1,14, \widehat{14}, 22)$ & 1 & 13 & 60 & 305 & $20.00 \%$ \\
$(\widehat{1}, 1,4,5,40)$ & 3 & 18 & 18 & 48 & $0.00 \%$ \\
$(1,1, \widehat{4}, 5,40)$ & 2 & 16 & 0 & 35 & $20.00 \%$ \\
$(1,1,4, \widehat{5}, 40)$ & 2 & 12 & 0 & 22 & $4.55 \%$ \\
$(\widehat{1}, 1,4,6,22)$ & 3 & 24 & 504 & 1016 & $9.55 \%$ \\
$(1,1, \widehat{4}, 6,22)$ & 1 & 24 & 78 & 1009 & $12.88 \%$ \\
$(1,1,4, \widehat{6}, 22)$ & 1 & 48 & 31 & 837 & $9.44 \%$ \\
$(\widehat{1}, 1,4,7,16)$ & 3 & 27 & 900 & 1476 & $21.00 \%$ \\
$(1, \widehat{4}, 7,16)$ & 1 & 20 & 196 & 1596 & $10.78 \%$ \\
$(\widehat{1}, 1,4,8,13)$ & 1 & 18 & 43 & 125 & $2.40 \%$ \\
$(1,1, \widehat{4}, 8,13)$ & 1 & 16 & 8 & 107 & $0.93 \%$ \\
$(1,1,4, \widehat{8}, 13)$ & 2 & 16 & 0 & 31 & $0.00 \%$ \\
$(1,1,4,8, \widehat{13})$ & 1 & 18 & 3 & 20 & $0.00 \%$ \\
$(\widehat{1}, 1,5,5,19)$ & 3 & 12 & 5 & 12 & $0.00 \%$ \\
$(1,1, \widehat{5}, 5,19)$ & 1 & 8 & 7 & 9 & $0.00 \%$ \\
$(1,1,5, \widehat{5}, 19)$ & 3 & 6 & 7 & 8 & $0.00 \%$ \\
$(\widehat{1}, 1,6,6,10)$ & 3 & 6 & 48 & 71 & $0.00 \%$ \\
$(1,1, \widehat{6}, 6,10)$ & 1 & 8 & 31 & 121 & $0.00 \%$ \\
\hline & & & & & Continued on next page \\
\hline
\end{tabular}


Table 3 - continued from previous page

\begin{tabular}{|c||c|c|c|c|c|}
\hline model & $\Delta$ & Max. & 3 family & $N$ fam. $(N>0)$ & Missing Mirrors \\
\hline$(\widehat{1}, 1,7,7,7)$ & 3 & 27 & 246 & 315 & $1.90 \%$ \\
$(\widehat{1}, 2,2,10,10)$ & 3 & 24 & 620 & 1060 & $5.38 \%$ \\
$(1, \widehat{2}, 2,10,10)$ & 1 & 25 & 764 & 5365 & $25.55 \%$ \\
$(1,2,2,2,2, \widehat{4})$ & 2 & 32 & 0 & 293 & $15.02 \%$ \\
$(\widehat{1}, 2,2,2,2,4)$ & 6 & 24 & 0 & 116 & $18.97 \%$ \\
$(1, \widehat{2}, 2,2,2,4)$ & 2 & 32 & 0 & 1499 & $11.41 \%$ \\
$(\widehat{1}, 2,2,6,22)$ & 3 & 24 & 136 & 423 & $9.46 \%$ \\
$(1, \widehat{2}, 2,6,22)$ & 1 & 18 & 204 & 1717 & $21.26 \%$ \\
$(1,2,2, \widehat{6}, 22)$ & 1 & 48 & 75 & 1010 & $16.93 \%$ \\
$(\widehat{1}, 2,2,7,16)$ & 3 & 12 & 3 & 17 & $0.00 \%$ \\
$(1, \widehat{2}, 2,7,16)$ & 1 & 12 & 0 & 61 & $6.56 \%$ \\
$(\widehat{1}, 2,3,3,58)$ & 1 & 12 & 57 & 203 & $2.96 \%$ \\
$(1, \widehat{2}, 3,3,58)$ & 1 & 16 & 31 & 245 & $2.04 \%$ \\
$(1,2, \widehat{3}, 3,58)$ & 1 & 12 & 54 & 493 & $10.34 \%$ \\
$(1,2,3,3, \widehat{58})$ & 1 & 24 & 0 & 52 & $0.00 \%$ \\
$(\widehat{1}, 2,3,4,18)$ & 2 & 12 & 0 & 24 & $0.00 \%$ \\
$(1, \widehat{2}, 3,4,18)$ & 2 & 24 & 0 & 76 & $0.00 \%$ \\
$(1,2, \widehat{3}, 4,18)$ & 2 & 24 & 0 & 103 & $3.88 \%$ \\
$(1,2,3, \widehat{4}, 18)$ & 2 & 16 & 0 & 51 & $0.00 \%$ \\
$(1, \widehat{2}, 4,4,10)$ & 1 & 24 & 419 & 3016 & $15.02 \%$ \\
$(1,2, \widehat{4}, 4,10)$ & 1 & 20 & 451 & 4097 & $22.19 \%$ \\
$(\widehat{1}, 2,4,6,6)$ & 6 & 12 & 0 & 18 & $11.11 \%$ \\
$(1, \widehat{2}, 4,6,6)$ & 2 & 24 & 0 & 172 & $8.14 \%$ \\
$(1,2, \widehat{4}, 6,6)$ & 4 & 16 & 0 & 35 & $8.57 \%$ \\
$(1,2,4, \widehat{6}, 6)$ & 2 & 48 & 0 & 305 & $14.75 \%$ \\
$(\widehat{1}, 3,3,3,13)$ & 1 & 18 & 125 & 692 & $3.18 \%$ \\
$(1, \widehat{3}, 3,3,13)$ & 1 & 36 & 444 & 3383 & $12.33 \%$ \\
$(1,3,3,3, \widehat{13})$ & 1 & 36 & 41 & 410 & $0.73 \%$ \\
$(\widehat{1}, 3,3,4,8)$ & 2 & 14 & 0 & 63 & $0.00 \%$ \\
$(1, \widehat{3}, 3,4,8)$ & 1 & 24 & 18 & 411 & $12.65 \%$ \\
$(1,3,3, \widehat{4}, 8)$ & 2 & 18 & 0 & 122 & $0.82 \%$ \\
$(1,3,3,4, \widehat{8})$ & 1 & 18 & 3 & 51 & $1.96 \%$ \\
$(1, \widehat{4}, 4,4,4)$ & 1 & 24 & 947 & 8018 & $12.91 \%$ \\
\hline & & & & & Continued on next page \\
\hline
\end{tabular}


Table 3 - continued from previous page

\begin{tabular}{|c||c|c|c|c|c|}
\hline model & $\Delta$ & Max. & 3 family & $N$ fam. $(N>0)$ & Missing Mirrors \\
\hline$(\widehat{1}, 5,42,922)$ & 6 & 24 & 0 & 8 & $0.00 \%$ \\
$(1, \widehat{5}, 42,922)$ & 1 & 28 & 0 & 15 & $0.00 \%$ \\
$(1,5, \widehat{42}, 922)$ & 2 & 44 & 0 & 18 & $0.00 \%$ \\
$(\widehat{1}, 5,43,628)$ & 1 & 18 & 5 & 19 & $5.26 \%$ \\
$(1, \widehat{5}, 43,628)$ & 1 & 24 & 6 & 27 & $7.41 \%$ \\
$(\widehat{1}, 5,46,334)$ & 3 & 24 & 5 & 50 & $2.00 \%$ \\
$(1, \widehat{5}, 46,334)$ & 1 & 28 & 8 & 67 & $0.00 \%$ \\
$(\widehat{1}, 5,47,292)$ & 6 & 24 & 0 & 8 & $0.00 \%$ \\
$(1, \widehat{5}, 47,292)$ & 1 & 28 & 0 & 7 & $0.00 \%$ \\
$(1,5, \widehat{4}, 292)$ & 3 & 12 & 3 & 5 & $0.00 \%$ \\
$(\widehat{1}, 5,52,187)$ & 6 & 12 & 0 & 5 & $0.00 \%$ \\
$(1, \widehat{5}, 52,187)$ & 1 & 16 & 0 & 15 & $20.00 \%$ \\
$(\widehat{1}, 5,54,166)$ & 3 & 30 & 0 & 51 & $0.00 \%$ \\
$(1, \widehat{5}, 54,166)$ & 1 & 36 & 13 & 68 & $1.47 \%$ \\
$(1,5, \widehat{54}, 166)$ & 1 & 20 & 0 & 43 & $0.00 \%$ \\
$(\widehat{1}, 5,58,138)$ & 1 & 12 & 2 & 26 & $0.00 \%$ \\
$(1, \widehat{5}, 58,138)$ & 1 & 16 & 6 & 33 & $0.00 \%$ \\
$(1,5,58,138)$ & 2 & 48 & 0 & 9 & $0.00 \%$ \\
$(\widehat{1}, 5,61,124)$ & 3 & 36 & 5 & 23 & $8.70 \%$ \\
$(1, \widehat{5}, 61,124)$ & 1 & 42 & 1 & 46 & $23.91 \%$ \\
$(\widehat{1}, 5,68,103)$ & 1 & 18 & 18 & 40 & $12.50 \%$ \\
$(1, \widehat{5}, 68,103)$ & 1 & 22 & 2 & 34 & $0.00 \%$ \\
$(\widehat{1}, 5,82,82)$ & 3 & 48 & 6 & 53 & $0.00 \%$ \\
$(1, \widehat{5}, 82,82)$ & 1 & 56 & 22 & 81 & $1.23 \%$ \\
$(\widehat{1}, 6,23,598)$ & 1 & 2 & 0 & 12 & $8.33 \%$ \\
$(1, \widehat{6}, 23,598)$ & 2 & 8 & 0 & 16 & $0.00 \%$ \\
$(1,6,23,598)$ & 2 & 12 & 0 & 50 & $8.00 \%$ \\
$(\widehat{1}, 6,24,310)$ & 6 & 12 & 0 & 11 & $18.18 \%$ \\
$(1, \widehat{6}, 24,310)$ & 1 & 32 & 10 & 104 & $0.96 \%$ \\
$(\widehat{1}, 6,25,214)$ & 3 & 12 & 12 & 24 & $0.00 \%$ \\
$(1, \widehat{6}, 25,214)$ & 2 & 32 & 0 & 57 & $8.77 \%$ \\
$(\widehat{1}, 6,26,166)$ & 6 & 6 & 0 & 14 & $0.00 \%$ \\
$(1, \widehat{6}, 26,166)$ & 2 & 24 & 0 & 128 & $0.78 \%$ \\
\hline & & & & & Continued on next page \\
\hline
\end{tabular}


Table 3 - continued from previous page

\begin{tabular}{|c||c|c|c|c|c|}
\hline model & $\Delta$ & Max. & 3 family & $N$ fam. $(N>0)$ & Missing Mirrors \\
\hline$(1,6, \widehat{26}, 166)$ & 1 & 10 & 6 & 70 & $2.86 \%$ \\
$(\widehat{1}, 6,28,118)$ & 1 & 20 & 236 & 1003 & $21.14 \%$ \\
$(1, \widehat{6}, 28,118)$ & 1 & 40 & 174 & 1531 & $24.62 \%$ \\
$(\widehat{1}, 6,30,94)$ & 3 & 12 & 88 & 168 & $26.19 \%$ \\
$(1, \widehat{6}, 30,94)$ & 1 & 40 & 43 & 483 & $19.46 \%$ \\
$(1,6, \widehat{30}, 94)$ & 1 & 32 & 49 & 615 & $15.93 \%$ \\
$(1, \widehat{6}, 31,86)$ & 2 & 4 & 0 & 5 & $0.00 \%$ \\
$(1,6,31, \widehat{86})$ & 6 & 12 & 0 & 5 & $0.00 \%$ \\
$(\widehat{1}, 6,34,70)$ & 3 & 9 & 24 & 40 & $10.00 \%$ \\
$(1, \widehat{6}, 34,70)$ & 1 & 48 & 35 & 471 & $6.16 \%$ \\
$(\widehat{1}, 6,38,58)$ & 1 & 11 & 32 & 243 & $4.12 \%$ \\
$(1, \widehat{6}, 38,58)$ & 1 & 24 & 59 & 438 & $7.31 \%$ \\
$(1,6,38, \widehat{8})$ & 2 & 42 & 0 & 79 & $11.39 \%$ \\
$(1, \widehat{6}, 40,54)$ & 2 & 32 & 0 & 101 & $3.96 \%$ \\
$(1,6,40, \widehat{54})$ & 2 & 24 & 0 & 28 & $0.00 \%$ \\
$(\widehat{1}, 6,46,46)$ & 3 & 12 & 101 & 163 & $21.47 \%$ \\
$(1, \widehat{6}, 46,46)$ & 1 & 64 & 69 & 867 & $21.34 \%$ \\
$(\widehat{1}, 7,17,340)$ & 6 & 6 & 0 & 2 & $0.00 \%$ \\
$(1,7, \widehat{17}, 340)$ & 6 & 12 & 0 & 3 & $0.00 \%$ \\
$(\widehat{1}, 7,18,178)$ & 1 & 12 & 31 & 120 & $0.83 \%$ \\
$(\widehat{1}, 7,19,124)$ & 3 & 9 & 26 & 34 & $8.82 \%$ \\
$(1,7, \widehat{19}, 124)$ & 3 & 18 & 4 & 16 & $0.00 \%$ \\
$(\widehat{1}, 7,20,97)$ & 3 & 6 & 4 & 5 & $0.00 \%$ \\
$(1,7, \widehat{20}, 97)$ & 4 & 8 & 0 & 3 & $0.00 \%$ \\
$(\widehat{1}, 7,22,70)$ & 3 & 15 & 121 & 207 & $18.84 \%$ \\
$(\widehat{1}, 7,25,52)$ & 3 & 18 & 50 & 80 & $2.50 \%$ \\
$(\widehat{1}, 7,28,43)$ & 1 & 10 & 60 & 142 & $2.11 \%$ \\
$(\widehat{1}, 7,34,34)$ & 3 & 24 & 123 & 209 & $1.91 \%$ \\
$(\widehat{1}, 8,14,238)$ & 1 & 12 & 56 & 256 & $16.02 \%$ \\
$(1, \widehat{8}, 14,238)$ & 1 & 14 & 12 & 93 & $4.30 \%$ \\
$(1,8, \widehat{1} 4,238)$ & 1 & 12 & 21 & 401 & $15.46 \%$ \\
$(1,8,14, \widehat{238})$ & 1 & 48 & 0 & 62 & $9.68 \%$ \\
$(\widehat{1}, 8,16,88)$ & 1 & 19 & 64 & 530 & $12.64 \%$ \\
\hline & & & & & Continued on next page \\
\hline
\end{tabular}


Table 3 - continued from previous page

\begin{tabular}{|c||c|c|c|c|c|}
\hline model & $\Delta$ & Max. & 3 family & $N$ fam. $(N>0)$ & Missing Mirrors \\
\hline$(1, \widehat{8}, 16,88)$ & 1 & 28 & 43 & 477 & $5.87 \%$ \\
$(\widehat{1}, 8,18,58)$ & 1 & 23 & 726 & 3734 & $6.86 \%$ \\
$(1, \widehat{8}, 18,58)$ & 1 & 32 & 333 & 2234 & $5.73 \%$ \\
$(1,8,18, \widehat{58})$ & 1 & 84 & 97 & 1248 & $7.05 \%$ \\
$(\widehat{1}, 8,22,38)$ & 1 & 12 & 12 & 152 & $0.66 \%$ \\
$(1, \widehat{8}, 22,38)$ & 2 & 10 & 0 & 38 & $0.00 \%$ \\
$(\widehat{1}, 8,28,28)$ & 1 & 36 & 1530 & 7738 & $17.19 \%$ \\
$(1, \widehat{8}, 28,28)$ & 1 & 56 & 584 & 4618 & $9.44 \%$ \\
$(\widehat{1}, 9,20,31)$ & 3 & 6 & 8 & 10 & $0.00 \%$ \\
$(1, \widehat{9}, 20,31)$ & 1 & 9 & 1 & 3 & $0.00 \%$ \\
$(1,9, \widehat{20}, 31)$ & 1 & 8 & 2 & 5 & $0.00 \%$ \\
$(\widehat{2}, 10,10,10)$ & 1 & 12 & 28 & 518 & $0.00 \%$ \\
$(\widehat{2}, 2,2,2,2,2)$ & 1 & 48 & 940 & 14131 & $8.90 \%$ \\
$(\widehat{2}, 2,2,3,18)$ & 1 & 24 & 75 & 1029 & $13.70 \%$ \\
$(2,2,2, \widehat{3}, 18)$ & 2 & 30 & 0 & 286 & $11.19 \%$ \\
$(\widehat{2}, 2,2,4,10)$ & 1 & 24 & 185 & 3216 & $13.50 \%$ \\
$(2,2,2, \widehat{4}, 10)$ & 2 & 24 & 0 & 534 & $17.42 \%$ \\
$(\widehat{2}, 2,2,6,6)$ & 1 & 24 & 383 & 9788 & $5.85 \%$ \\
$(2,2,2, \widehat{6}, 6)$ & 1 & 48 & 178 & 3288 & $6.60 \%$ \\
$(\widehat{2}, 2,3,3,8)$ & 1 & 16 & 23 & 443 & $1.35 \%$ \\
$(2,2, \widehat{3}, 3,8)$ & 1 & 24 & 55 & 1112 & $2.34 \%$ \\
$(2,2,3,3, \widehat{8})$ & 1 & 32 & 33 & 360 & $0.56 \%$ \\
$(2,2, \widehat{4}, 4,4)$ & 2 & 24 & 0 & 471 & $5.73 \%$ \\
$(\widehat{2}, 3,20,218)$ & 1 & 14 & 0 & 52 & $1.92 \%$ \\
$(2, \widehat{3}, 20,218)$ & 2 & 30 & 0 & 76 & $3.95 \%$ \\
$(2,3, \widehat{20}, 218)$ & 2 & 32 & 0 & 86 & $4.65 \%$ \\
$(\widehat{2}, 3,22,118)$ & 1 & 12 & 24 & 157 & $8.92 \%$ \\
$(2, \widehat{3}, 22,118)$ & 1 & 54 & 10 & 228 & $6.58 \%$ \\
$(\widehat{2}, 3,23,98)$ & 1 & 12 & 32 & 243 & $0.00 \%$ \\
$(2, \widehat{3}, 23,98)$ & 1 & 54 & 30 & 372 & $0.81 \%$ \\
$(2,3, \widehat{23}, 98)$ & 1 & 32 & 24 & 260 & $0.38 \%$ \\
$(\widehat{2}, 3,26,68)$ & 1 & 12 & 0 & 32 & $6.25 \%$ \\
$(2, \widehat{3}, 26,68)$ & 2 & 24 & 0 & 79 & $2.53 \%$ \\
\hline & & & & & Continued on next page \\
\hline
\end{tabular}


Table 3 - continued from previous page

\begin{tabular}{|c||c|c|c|c|c|}
\hline model & $\Delta$ & Max. & 3 family & $N$ fam. $(N>0)$ & Missing Mirrors \\
\hline$(\widehat{2}, 3,28,58)$ & 1 & 18 & 169 & 1340 & $3.96 \%$ \\
$(2, \widehat{3}, 28,58)$ & 1 & 78 & 308 & 3150 & $10.63 \%$ \\
$(2,3,28, \widehat{58})$ & 1 & 40 & 13 & 137 & $8.03 \%$ \\
$(\widehat{2}, 3,38,38)$ & 1 & 24 & 125 & 1038 & $14.84 \%$ \\
$(2, \widehat{3}, 38,38)$ & 1 & 108 & 166 & 2053 & $10.42 \%$ \\
$(\widehat{2}, 4,11,154)$ & 2 & 4 & 0 & 5 & $0.00 \%$ \\
$(2, \widehat{4}, 11,154)$ & 2 & 8 & 0 & 8 & $0.00 \%$ \\
$(2,4,13,58)$ & 1 & 8 & 62 & 228 & $4.39 \%$ \\
$(2, \widehat{4}, 13,58)$ & 1 & 12 & 27 & 306 & $12.42 \%$ \\
$(2,4, \widehat{13}, 58)$ & 2 & 30 & 0 & 95 & $2.11 \%$ \\
$(2,4,13, \widehat{58})$ & 1 & 32 & 0 & 19 & $15.79 \%$ \\
$(\widehat{2}, 4,14,46)$ & 1 & 12 & 0 & 57 & $8.77 \%$ \\
$(2, \widehat{4}, 14,46)$ & 1 & 12 & 2 & 29 & $10.34 \%$ \\
$(2,4, \widehat{14}, 46)$ & 2 & 12 & 0 & 68 & $0.00 \%$ \\
$(2, \widehat{4}, 16,34)$ & 1 & 8 & 8 & 248 & $9.27 \%$ \\
$(\widehat{2}, 4,18,28)$ & 1 & 12 & 18 & 161 & $0.00 \%$ \\
$(2, \widehat{4}, 18,28)$ & 1 & 10 & 0 & 79 & $2.53 \%$ \\
$(\widehat{2}, 4,22,22)$ & 1 & 12 & 0 & 61 & $0.00 \%$ \\
$(2, \widehat{4}, 22,22)$ & 1 & 8 & 2 & 24 & $0.00 \%$ \\
$(\widehat{2}, 5,10,40)$ & 3 & 12 & 4 & 19 & $0.00 \%$ \\
$(\widehat{2}, 5,12,26)$ & 1 & 12 & 22 & 141 & $0.71 \%$ \\
$(2,5, \widehat{12}, 26)$ & 1 & 5 & 10 & 40 & $0.00 \%$ \\
$(2,5,12, \widehat{2})$ & 1 & 15 & 4 & 20 & $10.00 \%$ \\
$(\widehat{2}, 5,8,138)$ & 1 & 6 & 2 & 69 & $8.70 \%$ \\
$(2, \widehat{5}, 8,138)$ & 1 & 6 & 1 & 35 & $2.86 \%$ \\
$(2,5, \widehat{8}, 138)$ & 1 & 6 & 4 & 10 & $0.00 \%$ \\
$(\widehat{2}, 6,10,22)$ & 1 & 15 & 19 & 426 & $2.11 \%$ \\
$(2, \widehat{6}, 10,22)$ & 1 & 12 & 24 & 248 & $0.40 \%$ \\
$(\widehat{2}, 6,14,14)$ & 1 & 20 & 0 & 138 & $2.17 \%$ \\
$(2, \widehat{6}, 14,14)$ & 1 & 32 & 32 & 224 & $3.12 \%$ \\
$(2,6, \widehat{14}, 14)$ & 1 & 18 & 67 & 350 & $5.71 \%$ \\
$(2,6,14, \widehat{14})$ & 1 & 18 & 55 & 348 & $2.87 \%$ \\
$(\widehat{2}, 6,7,70)$ & 1 & 6 & 0 & 20 & $10.00 \%$ \\
\hline & & & & & Continued on next page \\
\hline
\end{tabular}


Table 3 - continued from previous page

\begin{tabular}{|c||c|c|c|c|c|}
\hline model & $\Delta$ & Max. & 3 family & $N$ fam. $(N>0)$ & Missing Mirrors \\
\hline$(2, \widehat{6}, 7,70)$ & 2 & 2 & 0 & 11 & $9.09 \%$ \\
$(2,6,8,38)$ & 1 & 16 & 48 & 387 & $2.58 \%$ \\
$(2, \widehat{6}, 8,38)$ & 1 & 22 & 18 & 320 & $3.12 \%$ \\
$(2,6, \widehat{8}, 38)$ & 1 & 18 & 1 & 140 & $0.00 \%$ \\
$(\widehat{2}, 7,10,16)$ & 1 & 10 & 14 & 114 & $1.75 \%$ \\
$(\widehat{2}, 7,7,34)$ & 1 & 6 & 2 & 8 & $0.00 \%$ \\
$(\widehat{2}, 8,10,13)$ & 1 & 8 & 2 & 61 & $4.92 \%$ \\
$(2, \widehat{8}, 10,13)$ & 4 & 8 & 0 & 6 & $0.00 \%$ \\
$(2,8,10, \widehat{13})$ & 2 & 22 & 0 & 28 & $0.00 \%$ \\
$(\widehat{2}, 8,8,18)$ & 1 & 24 & 311 & 2310 & $1.90 \%$ \\
$(2, \widehat{8}, 8,18)$ & 1 & 24 & 395 & 3115 & $1.28 \%$ \\
$(\widehat{3}, 3,10,58)$ & 1 & 30 & 80 & 486 & $0.00 \%$ \\
$(3,3,10, \widehat{58})$ & 1 & 16 & 5 & 49 & $0.00 \%$ \\
$(\widehat{3}, 3,12,33)$ & 1 & 16 & 2 & 89 & $0.00 \%$ \\
$(3,3, \widehat{12}, 33)$ & 2 & 12 & 0 & 9 & $0.00 \%$ \\
$(\widehat{3}, 3,13,28)$ & 1 & 54 & 1034 & 6388 & $5.42 \%$ \\
$(3,3, \widehat{13}, 28)$ & 1 & 48 & 145 & 999 & $0.20 \%$ \\
$(\widehat{3}, 3,18,18)$ & 1 & 68 & 1802 & 12434 & $1.17 \%$ \\
$(\widehat{3}, 3,3,3,3)$ & 1 & 50 & 3189 & 25714 & $20.91 \%$ \\
$(\widehat{3}, 3,9,108)$ & 1 & 20 & 3 & 92 & $1.09 \%$ \\
$(3,3, \widehat{9}, 108)$ & 2 & 12 & 0 & 12 & $0.00 \%$ \\
$(\widehat{3}, 4,10,18)$ & 2 & 18 & 0 & 76 & $0.00 \%$ \\
$(3, \widehat{4}, 10,18)$ & 2 & 8 & 0 & 20 & $0.00 \%$ \\
$(\widehat{3}, 4,13,13)$ & 1 & 48 & 11 & 138 & $0.00 \%$ \\
$(3, \widehat{4}, 13,13)$ & 2 & 12 & 0 & 83 & $0.00 \%$ \\
$(3,4, \widehat{13}, 13)$ & 1 & 30 & 16 & 79 & $0.00 \%$ \\
$(\widehat{3}, 4,6,118)$ & 1 & 18 & 0 & 203 & $4.93 \%$ \\
$(3, \widehat{4}, 6,118)$ & 2 & 8 & 0 & 48 & $0.00 \%$ \\
$(3,4, \widehat{6}, 118)$ & 1 & 2 & 0 & 84 & $1.19 \%$ \\
$(\widehat{3}, 4,7,43)$ & 1 & 24 & 4 & 16 & $0.00 \%$ \\
$(3, \widehat{4}, 7,43)$ & 2 & 4 & 0 & 5 & $0.00 \%$ \\
$(3,4,8,28)$ & 1 & 48 & 340 & 2638 & $4.66 \%$ \\
$(3, \widehat{4}, 8,28)$ & 1 & 20 & 71 & 1116 & $1.43 \%$ \\
\hline & & & & & Continued on next page \\
\hline
\end{tabular}


Table 3 - continued from previous page

\begin{tabular}{|c||c|c|c|c|c|}
\hline model & $\Delta$ & Max. & 3 family & $N$ fam. $(N>0)$ & Missing Mirrors \\
\hline$(3,4, \widehat{8}, 28)$ & 1 & 32 & 86 & 644 & $1.24 \%$ \\
$(\widehat{3}, 5,5,68)$ & 1 & 24 & 0 & 27 & $0.00 \%$ \\
$(3, \widehat{5}, 5,68)$ & 1 & 8 & 9 & 13 & $0.00 \%$ \\
$(3,5, \widehat{5}, 68)$ & 1 & 6 & 9 & 17 & $0.00 \%$ \\
$(\widehat{3}, 6,6,18)$ & 2 & 28 & 0 & 158 & $0.63 \%$ \\
$(3, \widehat{6}, 6,18)$ & 1 & 8 & 18 & 99 & $0.00 \%$ \\
$(\widehat{3}, 8,8,8)$ & 1 & 72 & 3046 & 23592 & $4.80 \%$ \\
$(3, \widehat{8}, 8,8)$ & 1 & 32 & 3137 & 21945 & $3.20 \%$ \\
$(\widehat{4}, 4,10,10)$ & 1 & 20 & 45 & 420 & $2.86 \%$ \\
$(\widehat{4}, 4,5,40)$ & 1 & 12 & 0 & 64 & $3.12 \%$ \\
$(\widehat{4}, 4,6,22)$ & 1 & 14 & 4 & 260 & $5.38 \%$ \\
$(4,4, \widehat{6}, 22)$ & 4 & 8 & 0 & 26 & $3.85 \%$ \\
$(\widehat{4}, 4,7,16)$ & 1 & 18 & 104 & 491 & $3.87 \%$ \\
$(\widehat{4}, 4,8,13)$ & 1 & 12 & 5 & 166 & $1.81 \%$ \\
$(4,4, \widehat{8}, 13)$ & 4 & 16 & 0 & 23 & $0.00 \%$ \\
$(4,4,8, \widehat{13})$ & 2 & 28 & 0 & 25 & $0.00 \%$ \\
$(\widehat{4}, 5,5,19)$ & 4 & 8 & 0 & 2 & $0.00 \%$ \\
$(4, \widehat{5}, 5,19)$ & 1 & 8 & 1 & 2 & $0.00 \%$ \\
$(4,5, \widehat{5}, 19)$ & 3 & 3 & 1 & 1 & $0.00 \%$ \\
$(\widehat{4}, 6,6,10)$ & 2 & 2 & 0 & 2 & $0.00 \%$ \\
$(4, \widehat{6}, 6,10)$ & 4 & 8 & 0 & 9 & $0.00 \%$ \\
$(\widehat{4}, 7,7,7)$ & 2 & 6 & 0 & 14 & $0.00 \%$ \\
$(\widehat{5}, 5,5,12)$ & 1 & 8 & 13 & 82 & $0.00 \%$ \\
$(5, \widehat{5}, 5,12)$ & 3 & 6 & 29 & 40 & $0.00 \%$ \\
$(5,5,5, \widehat{12})$ & 1 & 8 & 6 & 22 & $0.00 \%$ \\
$(\widehat{6}, 6,6,6)$ & 1 & 16 & 174 & 1869 & $0.27 \%$ \\
$(1,10, \widetilde{11}, 154)$ & 2 & 12 & 0 & 23 & $0.00 \%$ \\
$(1,10, \widetilde{14}, 46)$ & 1 & 14 & 83 & 599 & $11.85 \%$ \\
$(1,1,1,1, \widetilde{5}, 40)$ & 6 & 12 & 0 & 5 & $20.00 \%$ \\
$(1,1,2, \widetilde{14}, 46)$ & 1 & 12 & 20 & 195 & $0.00 \%$ \\
$(1,1,4, \widetilde{5}, 40)$ & 6 & 12 & 0 & 13 & $0.00 \%$ \\
$(1, \widetilde{5}, 42,922)$ & 3 & 24 & 4 & 18 & $0.00 \%$ \\
$(1, \widetilde{5}, 43,628)$ & 1 & 24 & 6 & 24 & $4.17 \%$ \\
\hline & & & & & Continued on next page \\
\hline
\end{tabular}


Table 3 - continued from previous page

\begin{tabular}{|c||c|c|c|c|c|}
\hline model & $\Delta$ & Max. & 3 family & $N$ fam. $(N>0)$ & Missing Mirrors \\
\hline$(1, \widetilde{5}, 46,334)$ & 3 & 24 & 27 & 62 & $1.61 \%$ \\
$(1, \widetilde{5}, 47,292)$ & 3 & 24 & 2 & 7 & $0.00 \%$ \\
$(1, \widetilde{5}, 52,187)$ & 6 & 12 & 0 & 7 & $28.57 \%$ \\
$(1, \widetilde{5}, 54,166)$ & 3 & 30 & 27 & 61 & $0.00 \%$ \\
$(1, \widetilde{5}, 58,138)$ & 1 & 12 & 10 & 33 & $0.00 \%$ \\
$(1, \widetilde{5}, 61,124)$ & 3 & 36 & 10 & 19 & $0.00 \%$ \\
$(1, \widetilde{5}, 68,103)$ & 1 & 18 & 0 & 26 & $11.54 \%$ \\
$(1, \widetilde{5}, 82,82)$ & 3 & 48 & 39 & 74 & $1.35 \%$ \\
$(1,7, \widetilde{1}, 340)$ & 2 & 8 & 0 & 7 & $0.00 \%$ \\
$(1,8, \widetilde{14}, 238)$ & 1 & 12 & 16 & 428 & $23.13 \%$ \\
$(2,4, \widetilde{14}, 46)$ & 2 & 24 & 0 & 72 & $1.39 \%$ \\
$(2, \widetilde{5}, 8,138)$ & 1 & 8 & 2 & 40 & $0.00 \%$ \\
\hline \hline
\end{tabular}

\section{$6 \quad$ Results on Vector-like Particles}

As already emphasized before, we regard the chiral data (the number of families and the absence of chiral exotics) as the main area of study for these methods. However, we did also collect non-chiral data such as the number of mirrors, singlets and fractionally charged vector-like matter. The rationale for doing that is to determine how many distinct points in a given moduli space we obtain. Ideally, one could do that by comparing MIPFs. But this is hard to do. In principle any distinct simple current subgroup and/or any different choice of discrete torsion parameters defines a different MIPF, but there are usually large degeneracies, which are not all understood, and are difficult to "mod out" even if they are. So the simpler question we consider is: how many distinct massless spectra do we get (with "distinct" defined as in section 4). Obviously two identical massless spectra may still belong to different MIPFs, but when we made finer distinctions (as discussed in the next section) we found that in most cases a given spectrum yielded only one distinct refined spectrum. Hence it seems that our results give a pretty good estimate for the true number of distinct MIPFs.

Having gathered all this data we can also use them for a different purpose, namely to plot distributions of non-chiral quantities. The most important lesson that may be drawn from this procedure is how easy it would be to find exact RCFT spectra with certain desired properties, such as absence of certain vector-like exotics. Although this problem should really be addressed in a continuous geometric approach, as we have emphasized before, at least the existence of an exact RCFT example demonstrates that there are no fundamental obstacles for finding such solutions. 
In all of these plots we display on the vertical axis the number of distinct MIPFs, counting only models without chiral exotics, and with at least one family. We treat mirror symmetric MIPFs as distinct. Furthermore, all plots are stacked histograms: the contributions of the 8 different group types are stacked on top of each other for better visibility. In Figs. 9 and 10 we show respectively the distribution of $\mathrm{Q}$ and L-type mirrors. It is noteworthy that both distributions have shifted towards zero in comparison to the standard Gepner model case [1]. For Pati-Salami models, the Q mirror distribution peaks at 4 for standard Gepner models, and at 0 for lifted ones. For SM, Q $=\frac{1}{2}$ models the $Q$ mirrors peak at 3 (standard) and 0 (lifted). So in both cases the peak is at the MSSM value. For L mirror pair the MSSM value is one (the Higgs pair $H_{1}, H_{2}$ ); the peaks do indeed shift in that direction, from a value of around 20 to values of around 10 .

The analogous plots for $U$ and E mirrors are practically indistinguishable from those of Q mirrors, and the D-mirror plot is nearly identical to the L-mirror plot. This is because in $S U(5)$ GUTs, Q, U and E all come from the representation (10), while D, L both come from the $(\overline{5})$. Hence for the dominant contribution to the plots these distributions are identical. But even for the broken GUTs these quantities are not all unrelated: the spin-2 current $(3,1,10,0)$ of $S U(3) \times S U(2) \times U(1)_{30} \times U(1)_{20}$, that is always present in the halfinteger fractional charge models, relates $U$ and E. Since the half-integer fractional charge models (Pati-Salam and SM, $\mathrm{Q}=\frac{1}{2}$ ) dominate the statistics for the non-GUT models, there are only two differences that can still be non-trivial: D-L and Q-E. We show the distributions for these differences in Figs. 11 and 12 respectively. The main conclusion is that even in broken GUT models there is a tendency for mirror particles to be in complete GUT multiplets. This has the interesting consequence that mirror particles tend not to affect the convergence of couplings. The effect is somewhat weaker for members of the $(\overline{5})$, but it is still no entirely unreasonable to assume that the effect of mirrors on coupling unification could be small.

In Fig. 13 we show the distribution of fractionally charged particles. What is counted here along the $\mathrm{x}$-axis are half the number of $S U(3) \times S U(2) \times U(1)$ representations. The SM-dimensions (color triplets, weak doublets) of those representations are not taken into account, but the dimensions with respect to any additional gauge factor are counted as multiplicities. Most vector-like fractionally charged exotics come in pairs, but the halfinteger charged representation $(1,2,0)$ is real by itself (this is the only available $S U(3) \times$ $S U(2) \times U(1)$ representation, apart from the singlet, with that property). However, it too must appear with even multiplicities, since otherwise there would be an $S U(2)$ global anomaly (note that this anomaly cancel within each chiral family, and among the two members of any other vector-like pair). For this reason we have divided the number of fractionals by two, so that essentially what is shown is the number of vector-like pairs. There is an interesting structure visible in these plots. There are considerably more SM, $\mathrm{Q}=\frac{1}{2}$ models where the number of vector-like pairs is even. For the Pati-Salam models this goes even further, with a successive enhancement if the number of pairs is even, a multiple of four and a multiple of eight. The origin of this phenomenon is not clear.

The singlet distribution is shown in Fig. 14. The positions of the peaks of these distributions are not very different from those of the standard Gepner model case [1], but 
the distributions are much broader.

One might have hoped that weight lifting, since it increases more conformal weights than it decreases, would lower the number of singlets (as indeed it seems to do for the mirrors). However, one should keep in mind that not only the conformal weight changes, but that also the ground state dimensions in general increase, because the ground states are often in non-trivial representations of the extra non-abelian gauge groups of the lifted CFT. When counting states we did take these dimensions into account. Therefore the actual number of singlet particle representations is considerably smaller. This effect is more important for standard model singlets than for mirrors, because the latter get a contribution to their conformal weight from the standard model representation they carry, so that there is less "space" left for a non-trivial representation of a non-abelian factor: the total conformal weight has to add up to 1.

\section{Non-abelian Group Representations}

In orientifold models, matter that is charged under additional gauge group factors but not under the standard model is usually not counted as a singlet, but as hidden sector matter. One can perform a similar count here. In Fig. 15 we plot the distribution of standard model matter singlets (all singlets except the dilaton multiplet) that are in the trivial representation of the non-abelian factors of the "hidden sector". Here we are only considering non-abelian groups originating from the lifts. In general there will also be a few cases where the chiral algebra of the internal sector of the theory (that is, the part of the CFT outside $S O(10)$ ) is extended to a non-abelian group, but these extensions were not taken into account. As expected, the distributions move towards zero in comparison to Fig. 14, and there are even some cases where zero is reached, so that all standard model matter singlets are in a non-trivial representation of some non-abelian hidden sector group.

The latter are examples of chiral heterotic spectra where the only gauge singlets are the dilaton and the axion, $B_{\mu \nu}$ (and of course the graviton). However, this does not mean that there are no moduli, apart from the dilaton multiplet. One can observe the same phenomenon in enhanced symmetry points in the moduli space of Narain compactifications. In that case the moduli are in non-trivial representations of non-abelian groups, and giving them a v.e.v. breaks the gauge symmetry. It is likely that the same happens here and that these models correspond to enhanced symmetry points in a larger moduli space. On the other hand, if most or all of the moduli are in non-trivial non-abelian gauge group representations, this opens the way to dynamical mechanisms to lift them when these gauge interactions become strong. Note that even if standard model singlets are also singlets with respect to all non-abelian groups originating from the lift, they will usually still be charged under abelian factors in the internal sector. Indeed, in standard Gepner models most singlets (and in particular most moduli) are charged. In this case we know from comparison with the geometric case that moving in moduli space will break some or all of the $U(1)$ 's. For this reason we ignored such $U(1)$ charges when counting singlets. 
Analogously, one may ask if the fractionally charged matter could be mostly in nonabelian gauge representations. Indeed, one way around the problem of the fractionally charged particles would be that all of them are confined by some additional gauge force into integer charged particles. Although this looks like an ugly and far-fetched solution (and probably is) to a problem that is beautifully solved by field-theoretic $S U(5)$ GUTs, remarkably the first example of heterotic weight lifting we worked out in detail in [11] had precisely that feature. In that example, occurring in the tensor product $(3, \widehat{8}, 8,8)$, the gauge group contains a factor $A_{1,8}(S U(2)$ level 8), and in the massless spectrum all half-integer charged particles turned out to be in half-integer spin representations of this $S U(2)$, whereas all integer-charged particles are in integer spin representations (this was only checked for the massless spectrum).

In the present work we have merely checked if in general the fractionally charged matter is in non-trivial representations of non-abelian groups. The answer turns out to be mostly negative (even if it had been positive, this would still not guarantee that the gauge group confines them). The result is shown in Fig. 16. These distributions show, for all six types of broken GUTs, the number of MIPFs (y-axis) with a certain number of vector-like pairs of fractionally charged particles that are singlets with respect to the extra non-abelian factors. Clearly in the majority of cases there are at least some fractionally charged particles that are singlets of all non-abelian hidden sector groups, and hence cannot be confined. Interestingly, for the half-integer charge models there are large peaks at zero, which are completely absent for the third- and sixth integer charge models (of which there are very few; please note that the scales of these plots are very different). For the models contributing to these peaks all fractionally charged particles couple to non-abelian groups. However, as already remarked above, this still does not mean that they are confined, and anyhow this is only a small part of the total surface area.

Finally, one may ask if the standard model families couple to the additional nonabelian groups. If they do, and if those non-abelian groups remain unbroken, this will almost certainly give rise to serious phenomenological problems. For example, in the aforementioned model presented in [11] some of the standard model matter is in triplets of $S U(2)_{8}$, in such a way that some Yukawa couplings are forbidden. Here we have simply checked which fraction of the total amount of matter (families and mirror pairs) is fully abelian in the extra gauge groups. The result is shown in Fig. 17. There is a huge peak at $100 \%$, representing spectra for which all matter is in the singlet representation of the extra non-abelian groups. Many of the other spectra are not yet ruled out though: there will also be examples where only the mirror fermions couple to the extra gauge groups. This is not a problem and might even be a benefit.

However, we have not investigated such more detailed questions. The most important issue is whether the extra non-abelian groups introduced by heterotic weight lifting give rise to such serious obstacles that the whole class must be rejected. This seems not to be the case. 


\section{Conclusions}

We have studied a new region of the heterotic landscape that is a bit more genuinely $(0,2)$ than standard Gepner models. This immediately solves an annoying problem of the latter: the difficulty to get three families. Other classes we have studied (and which will be published in future work) seem to confirm this. If indeed the distributions we get for these models are typical for heterotic strings, then the observed number of families is not an issue of concern. The family distribution is fairly smooth around three families and does not have the sharp dip observed in orientifold models. Nevertheless, from these two different results in two accessible corners of the string landscape no conclusion can be drawn about such distributions on the full landscape.

What does remain an issue of concern is the occurrence of fractional charges. Unless in generic heterotic vacua vector-like particles are lifted to scales far above the weak scale, we have not found any convincing reason for their absence or extremely small abundance in our environment. In the class we studied here, as well as those studied in [1] and [32], fractionally charged particles are always present in the massless spectrum, with very few exceptions, and usually not all of them are coupled to other non-abelian interactions to confine them. The fact that examples can be found where they are absent does not change the fact that string theory seems to predict them.

Our results demonstrate the existence of hundreds of novel heterotic "mini-landscapes" where the standard model might be located, as shown in Table 3. Further study of the feasibility of this class require methods beyond RCFT. In particular, a geometric understanding of this class would be most welcome.

Presumably all this is just the tip of a huge iceberg. Along the same lines, one could still study weight lifting for exceptional $N=2$ MIPFs. Indeed, combining these two ideas is completely straightforward, but we have not done this yet. One can also construct internal sectors of heterotic strings partly out of free fermions and partly out of lifted Gepner models. This also yields new examples of three family models [35]. Another possibility is to use double lifts, as explained in [11] (so far a partial investigation did not yield examples with three families).

But the most important reason to think that there may be a large number of other possibilities is that the list of lifted Gepner models is not known to be complete, and presumably only contains the simplest possibilities, those that can be obtained by fairly straightforward manipulations of affine Lie algebras.

\section{Acknowledgements:}

This work has been partially supported by funding of the Spanish Ministerio de Ciencia e Innovación, Research Project FPA2008-02968, and by the Project CONSOLIDERINGENIO 2010, Programme CPAN (CSD2007-00042). The work of A.N.S. has been 
performed as part of the program FP 57 of Dutch Foundation for Fundamental Research of Matter (FOM).

\section{References}

[1] B. Gato-Rivera and A. N. Schellekens, arXiv:1003.6075 [hep-th].

[2] D. Gepner, Nucl. Phys. B 296 (1988) 757.

[3] A. N. Schellekens and S. Yankielowicz, Nucl. Phys. B 330 (1990) 103.

[4] T. P. T. Dijkstra, L. R. Huiszoon and A. N. Schellekens, Phys. Lett. B 609 (2005) 408; Nucl. Phys. B 710 (2005) 3.

[5] F. Gmeiner, R. Blumenhagen, G. Honecker, D. Lüst and T. Weigand, JHEP 0601, 004 (2006).

[6] X. G. Wen and E. Witten, Nucl. Phys. B 261 (1985) 651.

[7] G. G. Athanasiu, J. J. Atick, M. Dine and W. Fischler, Phys. Lett. B 214 (1988) 55.

[8] A. N. Schellekens, Phys. Lett. B 237 (1990) 363.

[9] D. Gepner, arXiv:hep-th/9301089.

[10] J. Fuchs, A. Klemm, C. Scheich and M. G. Schmidt, Annals Phys. 204 (1990) 1.

[11] B. Gato-Rivera and A. N. Schellekens, Nucl. Phys. B 828, 375 (2010) arXiv:0910.1526 [hep-th]].

[12] R. Blumenhagen and A. Wisskirchen, Nucl. Phys. B 454, 561 (1995) arXiv:hepth/9506104.

[13] A. D. Linde, Eternally Existing Self-reproducing Chaotic Inflationary Universe, Phys. Lett. B 175 (1986) 395.

[14] L. Susskind, "The anthropic landscape of string theory", In Carr, Bernard (ed.): Universe or Multiverse?, 247-266; arXiv:hep-th/0302219].

[15] Bert Schellekens, "Naar een waardig slot", 16 September 1998, ISBN 90-9012073-4 (In Dutch, English translation in arXiv:physics/0604134).

[16] A.N. Schellekens, “The Emperor's Last Clothes?", Rept. Prog. Phys. 71, 072201 (2008). A much extended version can be found in [arXiv:0807.3249 [physics.pop-ph]].

[17] K. R. Dienes and M. Lennek, Phys. Rev. D 80, 106003 (2009) arXiv:0809.0036 [hep-th]]. 
[18] A. E. Faraggi, Phys. Rev. D 46 (1992) 3204.

[19] W. Buchmuller, K. Hamaguchi, O. Lebedev and M. Ratz, Phys. Rev. Lett. 96 (2006) 121602 arXiv:hep-ph/0511035.

[20] O. Lebedev, H. P. Nilles, S. Raby, S. Ramos-Sanchez, M. Ratz, P. K. S. Vaudrevange and A. Wingerter, Phys. Lett. B 645 (2007) 88 arXiv:hep-th/0611095.

[21] O. Lebedev, H. P. Nilles, S. Ramos-Sanchez, M. Ratz and P. K. S. Vaudrevange, Phys. Lett. B 668 (2008) 331 [arXiv:0807.4384 [hep-th]].

[22] M. L. Perl, E. R. Lee and D. Loomba, Ann. Rev. Nucl. Part. Sci. 59 (2009) 47.

[23] A. N. Schellekens, Commun. Math. Phys. 153 (1993) 159 arXiv:hep-th/9205072.

[24] D. J. Gross, J. A. Harvey, E. J. Martinec and R. Rohm, Phys. Rev. Lett. 54, 502 (1985).

[25] P. Candelas, G. T. Horowitz, A. Strominger and E. Witten, Nucl. Phys. B 258 (1985) 46.

[26] W. Lerche, D. Lüst and A. N. Schellekens, Nucl. Phys. B 287 (1987) 477.

[27] A. N. Schellekens and N. P. Warner, Nucl. Phys. B 287 (1987) 317.

[28] M. Kreuzer and A. N. Schellekens, Nucl. Phys. B 411 (1994) 97 arXiv:hepth/9306145.

[29] E. Witten, Nucl. Phys. B 258 (1985) 75.

[30] B. Assel, K. Christodoulides, A. E. Faraggi, C. Kounnas and J. Rizos, Phys. Lett. B 683, 306 (2010) arXiv:0910.3697 [hep-th]].

[31] J. Rizos, arXiv:1003.0458 [hep-th].

[32] B. Assel, K. Christodoulides, A. E. Faraggi, C. Kounnas and J. Rizos, arXiv:1007.2268 [hep-th].

[33] L. B. Anderson, J. Gray, Y. H. He and A. Lukas, JHEP 1002 (2010) 054 arXiv:0911.1569 [hep-th]].

[34] M. Blaszczyk, S. G. Nibbelink, M. Ratz, F. Ruehle, M. Trapletti and P. K. S. Vaudrevange, Phys. Lett. B 683 (2010) 340 [arXiv:0911.4905 [hep-th]].

[35] M. Netjes, Master thesis, RU Nijmegen, August 2010. 


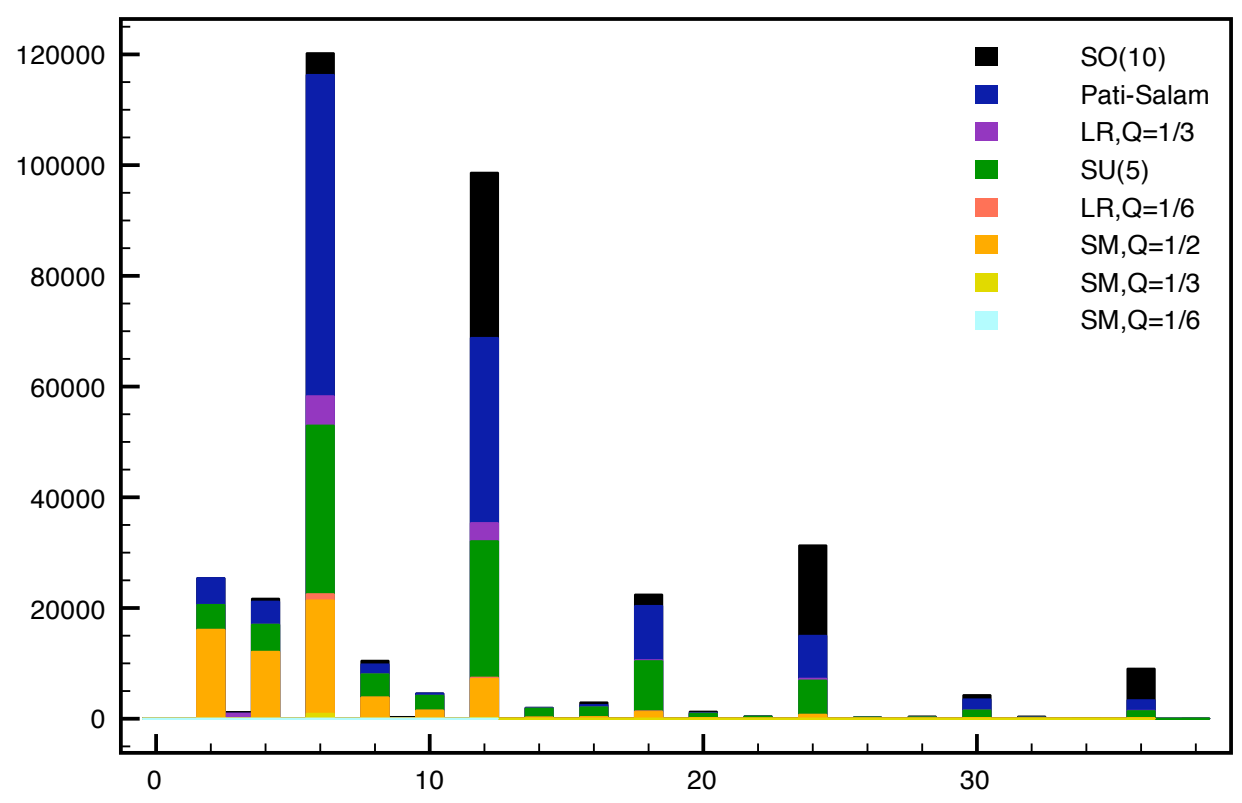

Figure 5: Family distribution for standard Gepner models.

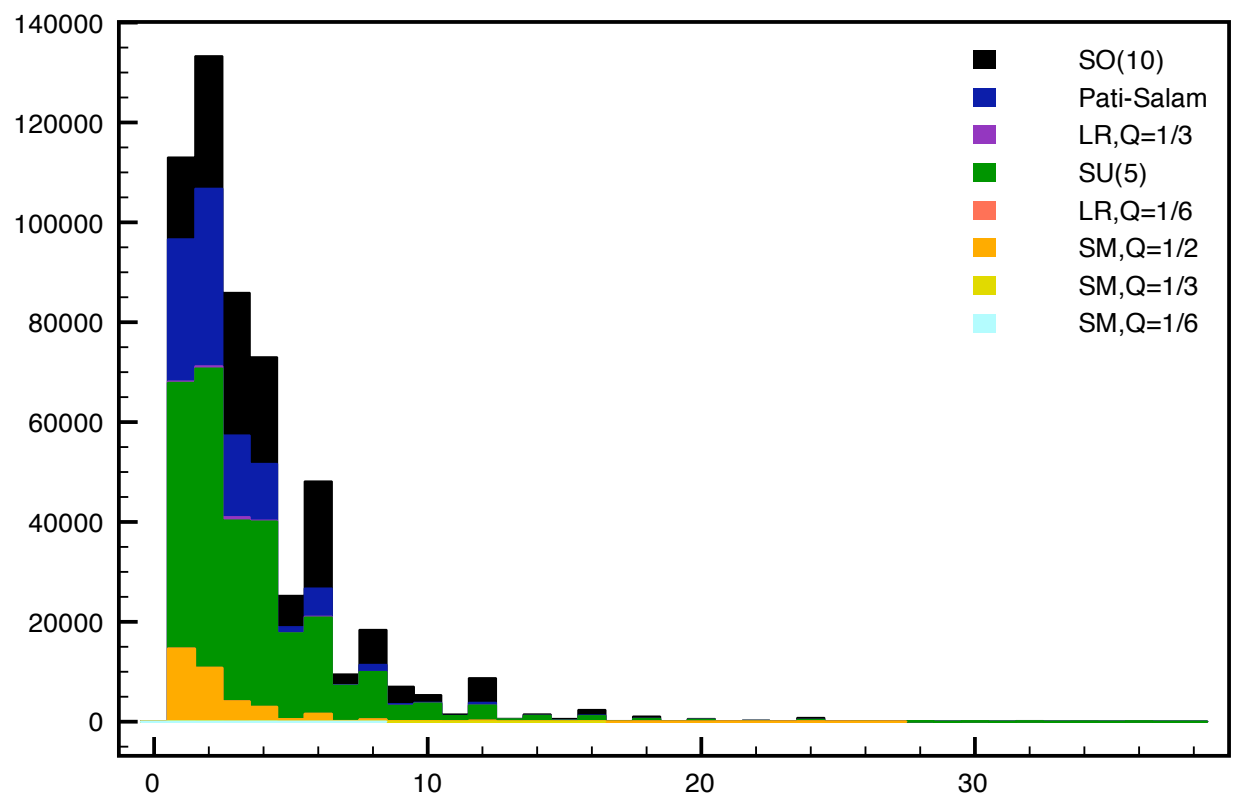

Figure 6: Family distribution for lifted Gepner models. 


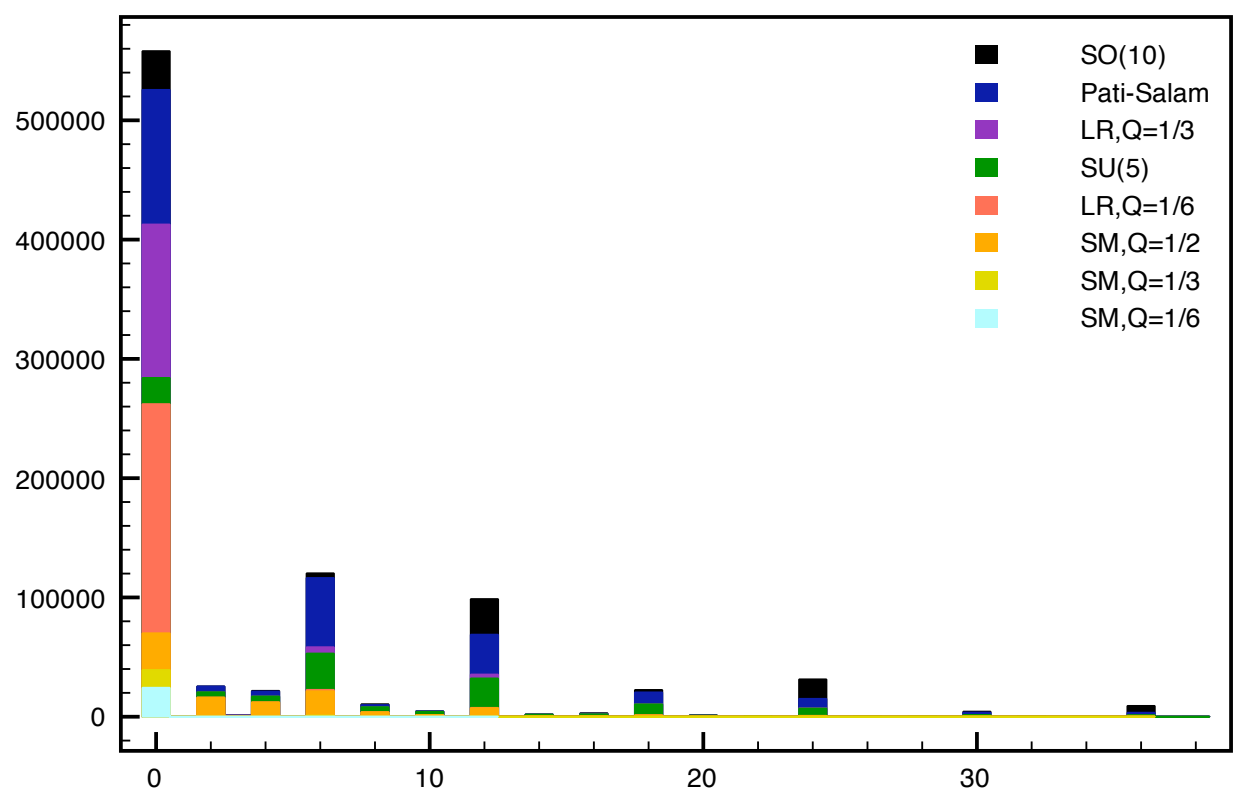

Figure 7: Family distribution for standard Gepner models, including zero families

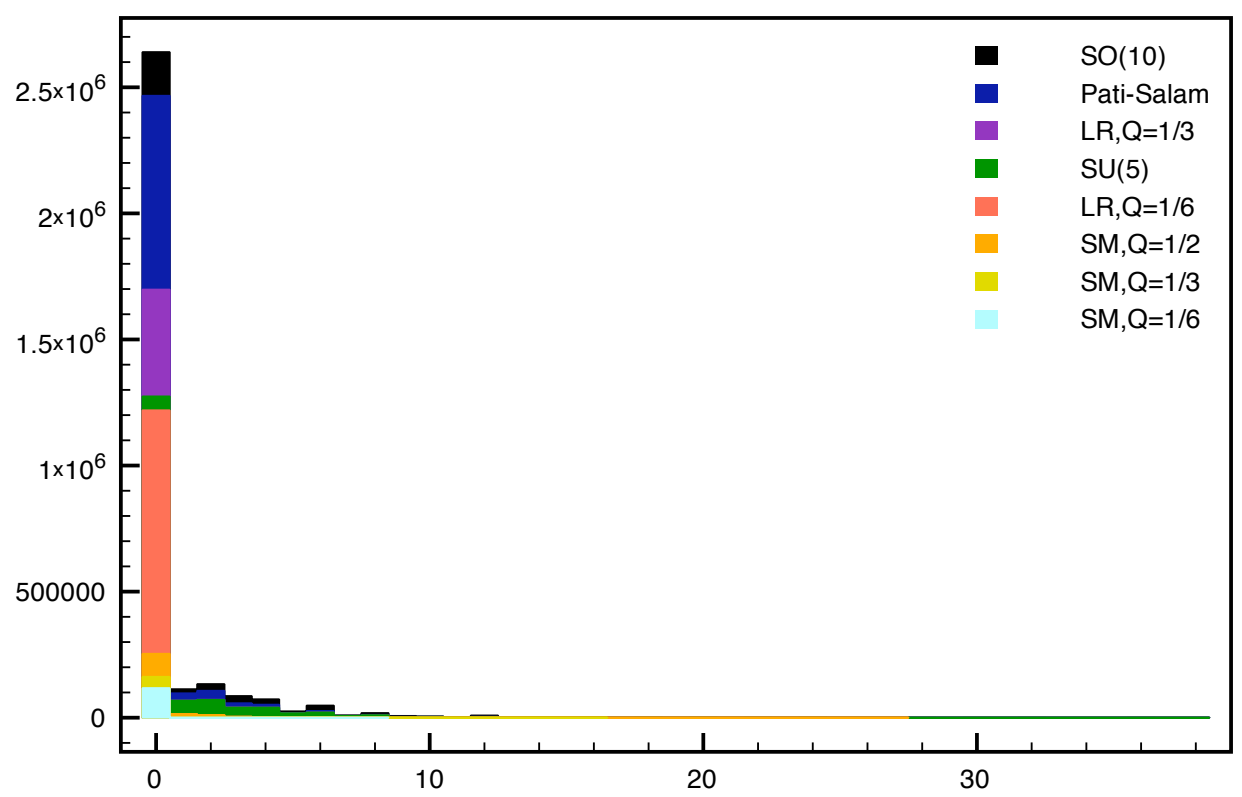

Figure 8: Family distribution for lifted Gepner models, including zero families. 


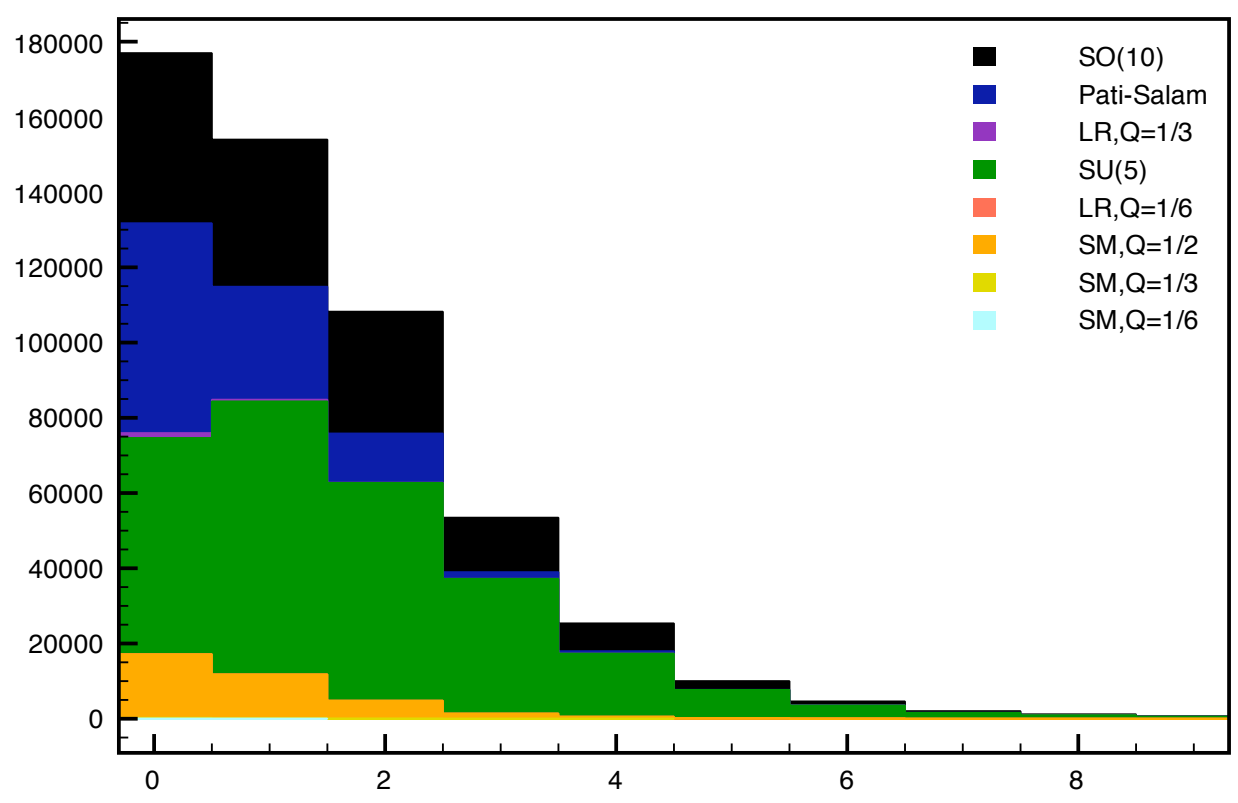

Figure 9: Q-Mirror distribution.

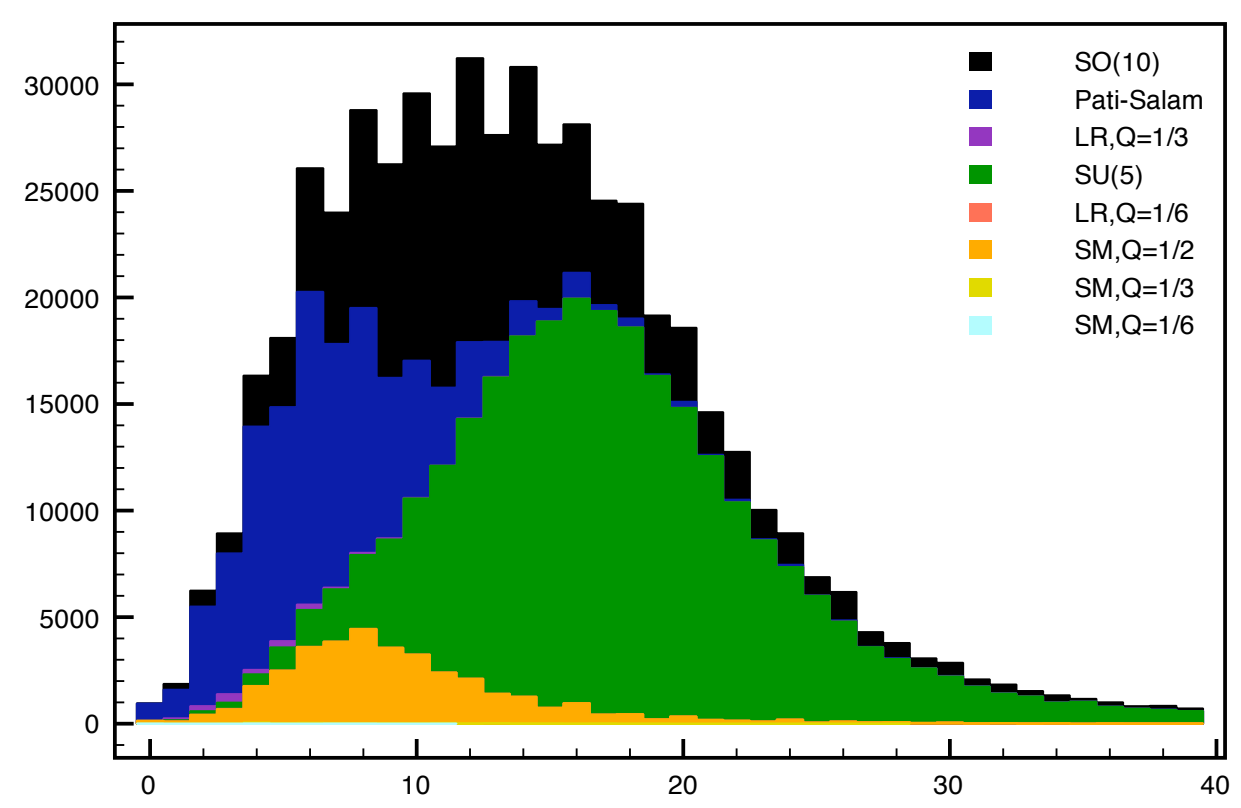

Figure 10: L-Mirror distribution. 


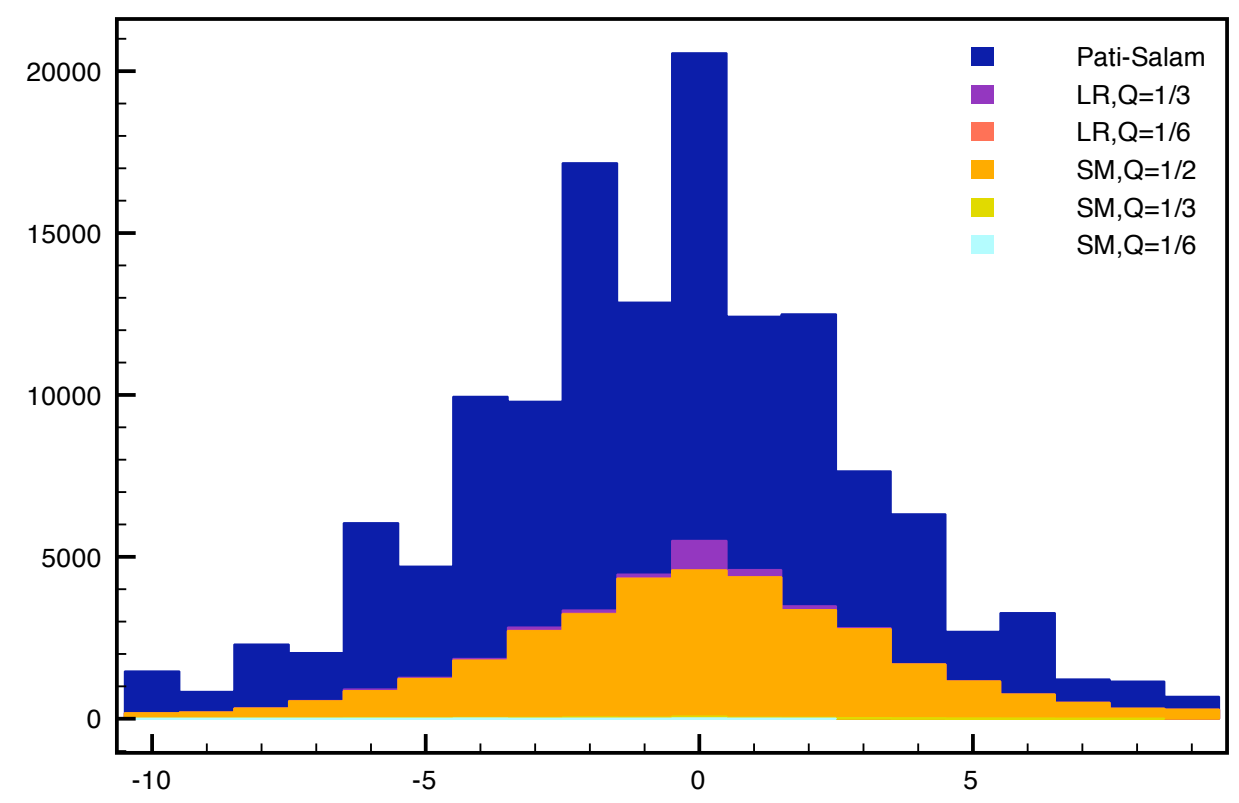

Figure 11: Difference between the D and L distributions.

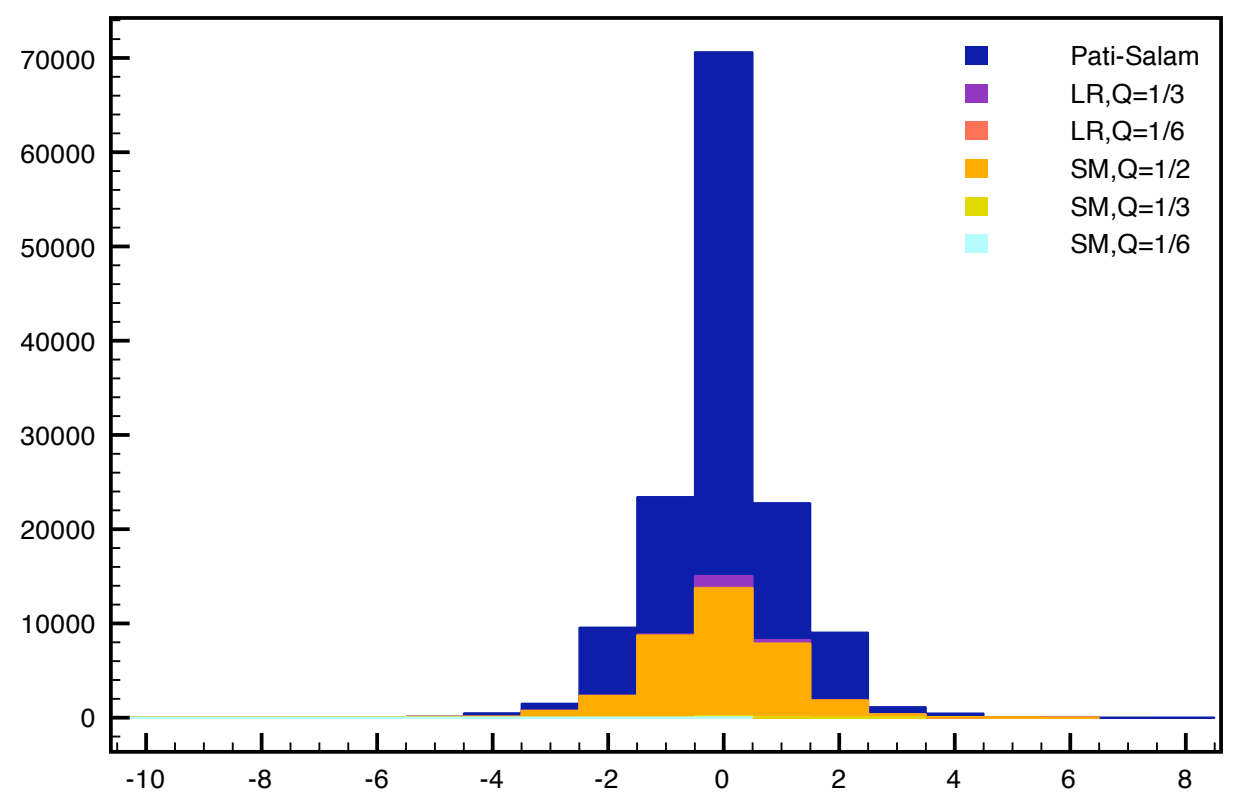

Figure 12: Difference between the $\mathrm{Q}$ and $\mathrm{E}$ distributions. 


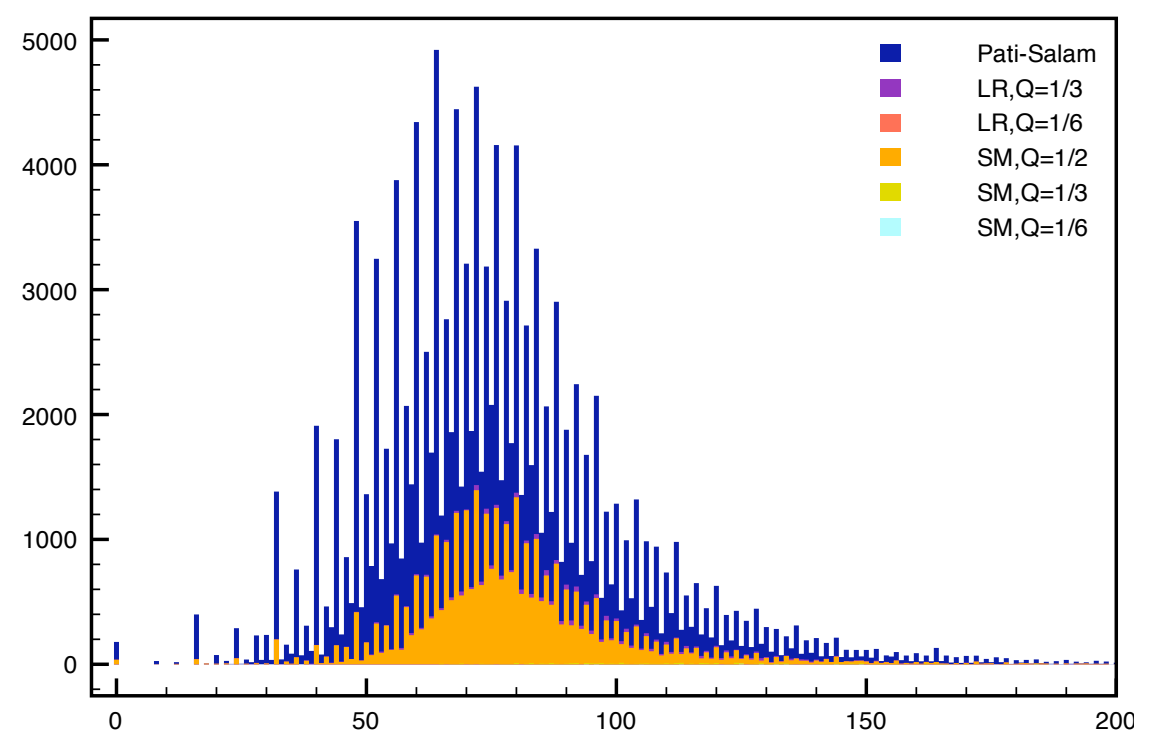

Figure 13: The number of MIPFs with a certain number of vector-like pairs of fractionally charged particles.

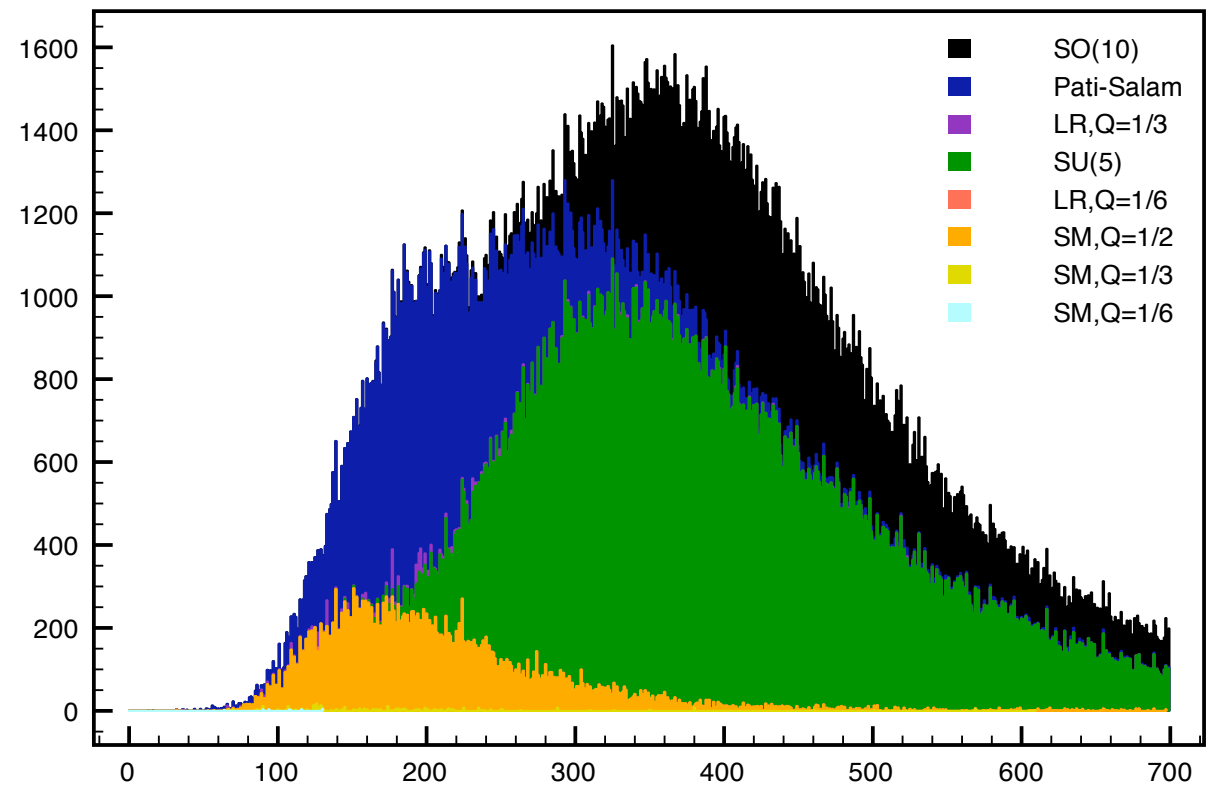

Figure 14: Singlet distribution. 


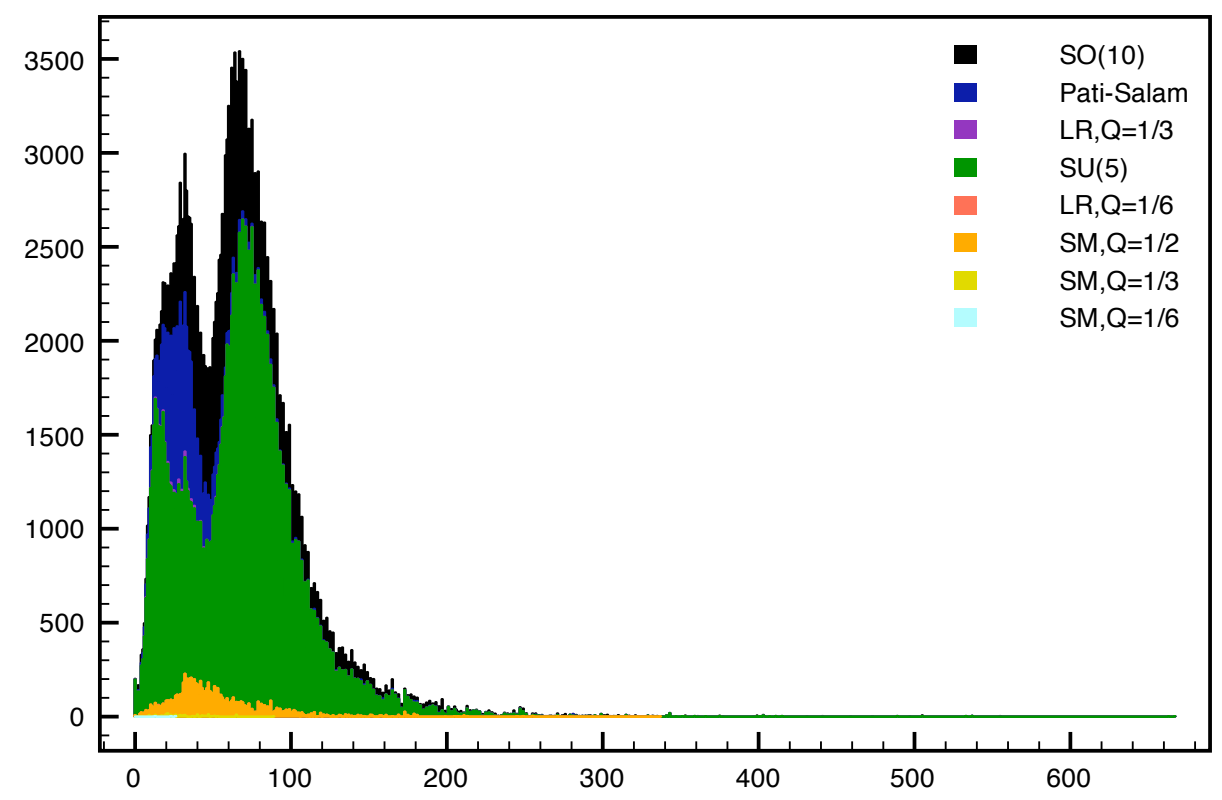

Figure 15: Abelian singlet distribution: singlets that do not couple to non-abelian hidden sector groups. 

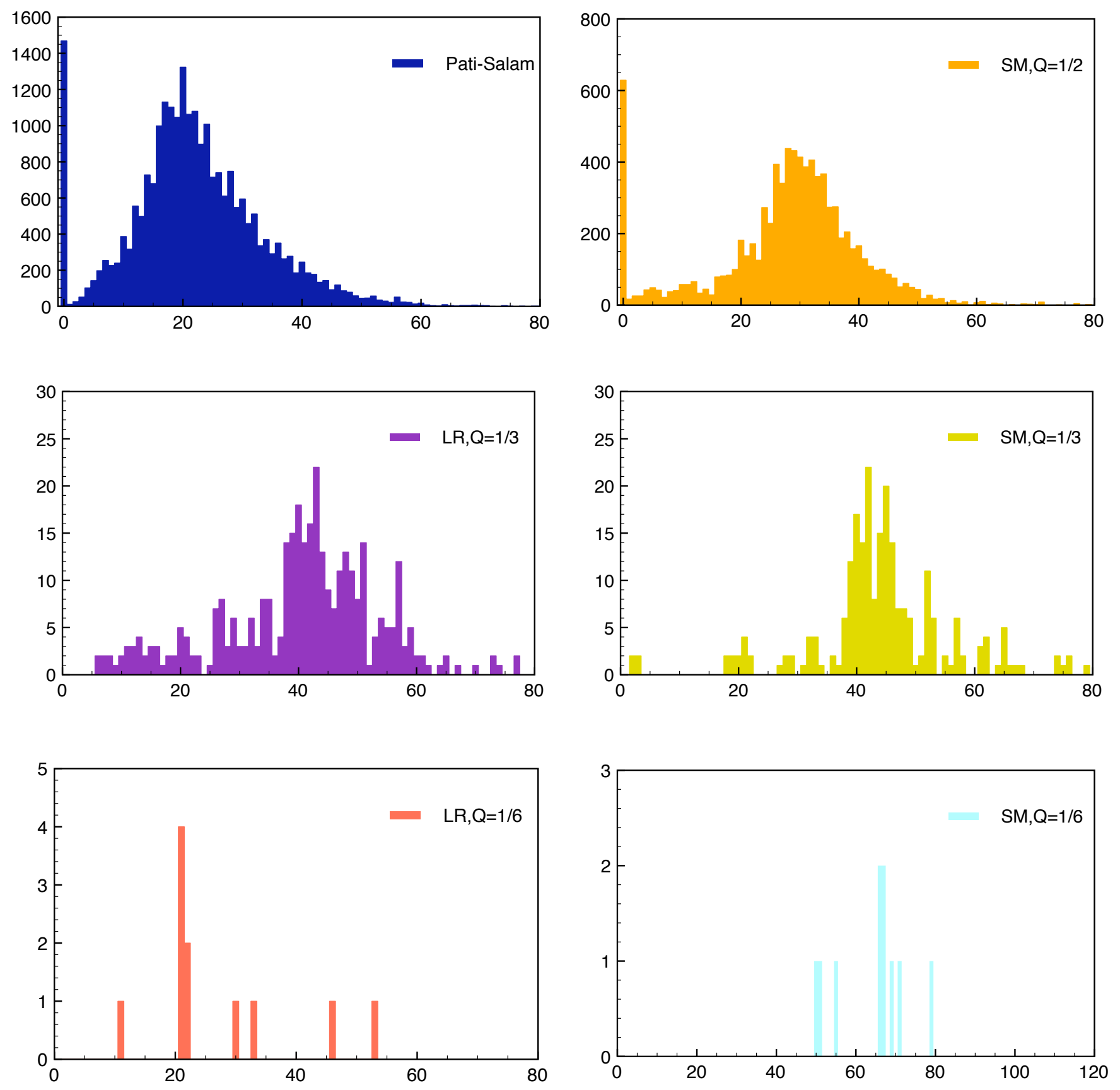

Figure 16: Abelian fractional charge distributions. 


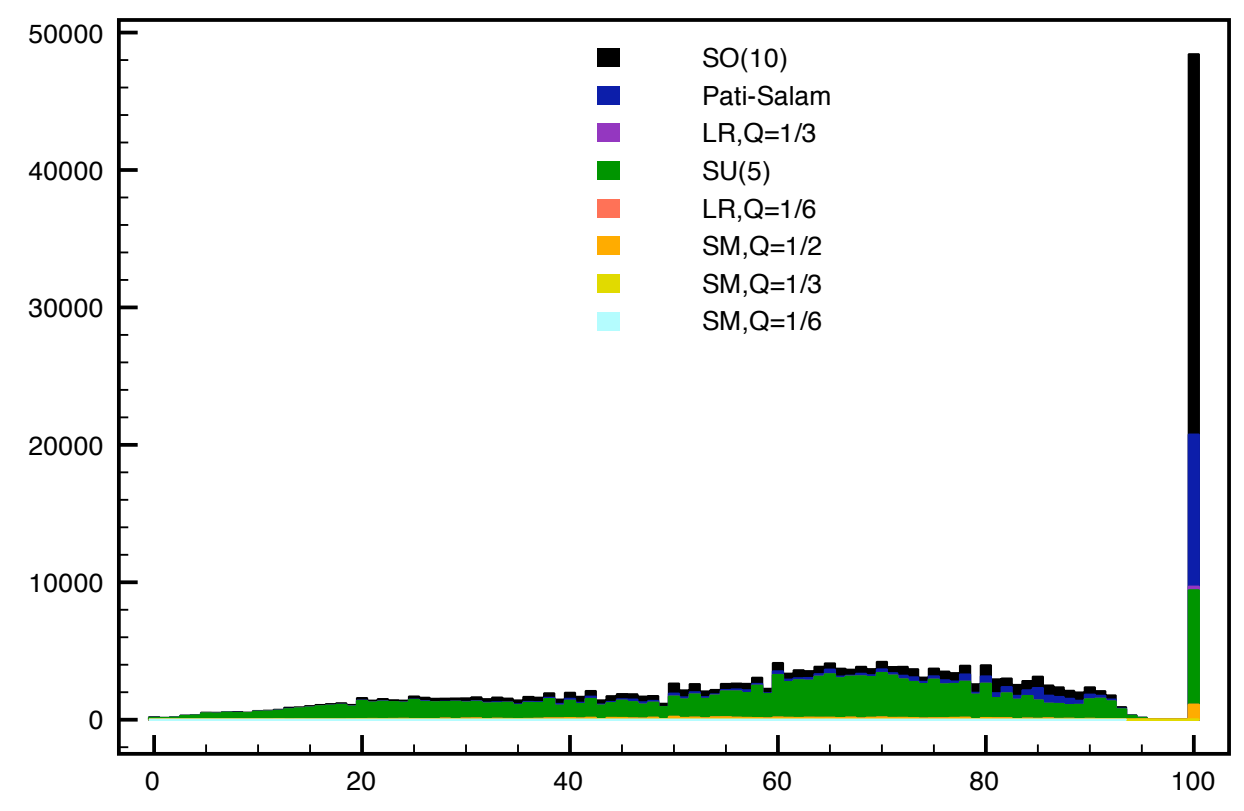

Figure 17: Percentage of all matter that is abelian with respect to the extra gauge factors. 